\title{
An innovative, highly sensitive receiver system for the Square Kilometre Array Mid radio telescope
}

\author{
Gie Han Tan ${ }^{* a}$, Robert Lehmensiek ${ }^{\mathrm{b}}$, Bhushan Billade ${ }^{\mathrm{c}}$, Krzysztof Caputa $^{\mathrm{d}}$, Stéphane Gauffre ${ }^{\mathrm{e}, \mathrm{f}}$, Isak \\ P. Theron ${ }^{b}$, Miroslav Pantaleev ${ }^{c}$, Zoran Ljusic ${ }^{\mathrm{d}}$, Benjamin Quertier, ${ }^{\mathrm{e}}$, Adriaan Peens - Hough ${ }^{\mathrm{g}}$ \\ ${ }^{\text {a }}$ SKA Organisation, Jodrell Bank Observatory, Lower Withington, Macclesfield, Cheshire,

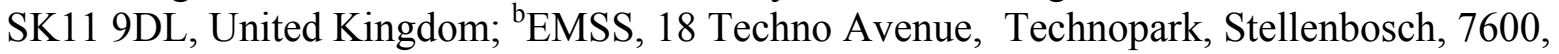 \\ SouthAfrica; 'Onsala Space Observatory, Observatorievägen 90, Råö, SE-439 92 Onsala, Sweden; \\ ${ }^{\mathrm{d}}$ National Research Council, Box 248, 717 White Lake Road, Penticton, BC V2A 6J9, Canada; \\ ${ }^{\mathrm{e}}$ Univ. Bordeaux, LAB, UMR 5804, F-33615, Pessac, France; ${ }^{\mathrm{f}} \mathrm{CNRS}$, LAB, UMR 5804, F-33615, \\ Pessac, France; ${ }^{g}$ SKA South Africa, $3{ }^{\text {rd }}$ Floor, The Park, Park Road, Pinelands, 7405, \\ South Africa
}

\begin{abstract}
The Square Kilometre Array (SKA) Project is a global science and engineering project realizing the next-generation radio telescopes operating in the metre and centimetre wavelengths regions. This paper addresses design concepts of the broadband, exceptionally sensitive receivers and reflector antennas deployed in the SKA1-Mid radio telescope to be located in South Africa. SKA1-Mid ( $350 \mathrm{MHz}-13.8 \mathrm{GHz}$ with an option for an upper limit of $\sim 24 \mathrm{GHz}$ ) will consist of 133 reflector antennas using an unblocked aperture, offset Gregorian configuration with an effective diameter of $15 \mathrm{~m}$.

Details on the unblocked aperture Gregorian antennas, low noise front ends and advanced direct digitization receivers, are provided from a system design perspective. The unblocked aperture results in increased aperture efficiency and lower side-lobe levels compared to a traditional on-axis configuration. The low side-lobe level reduces the noise contribution due to ground pick-up but also makes the antenna less susceptible to ground-based RFI sources. The addition of extra shielding on the sub-reflector provides a further reduction of ground pick-up. The optical design of the SKA1-Mid reflector antenna has been tweaked using advanced EM simulation tools in combination with sophisticated models for sky, atmospheric and ground noise contributions. This optimal antenna design in combination with very low noise, partially cryogenic, receivers and wide instantaneous bandwidth provide excellent receiving sensitivity in combination with instrumental flexibility to accommodate a wide range of astronomical observation modes.
\end{abstract}

Keywords: Square Kilometre Array, SKA, radio telescope, receiver, reflector antenna, radio astronomy, optimisation methods

\section{INTRODUCTION}

The Square Kilometre Array (SKA) project is a global science and engineering project aiming at designing and constructing the next-generation radio telescope operating in the metre and centimetre wavelengths regions ${ }^{1}$. The first phase of construction of the telescope (SKA1), which represents roughly $10 \%$ of the full SKA (SKA2), advances well with its design and is expected to be operational early next decade. The SKA radio telescopes will be co-hosted in two locations with central array sites in Australia, SKA1-Low (50 - $350 \mathrm{MHz})$, and South Africa, SKA1-Mid (350 MHz $13.8 \mathrm{GHz}$ with an option for an upper limit of $\sim 24 \mathrm{GHz}$ ).

\subsection{SKA Science}

The proposal for the SKA arose from a scientific demand from the community for new capabilities to address fundamental questions in astronomy. An open scientific consultation process, leading to a list of Science Drivers - key areas of science that the SKA will enable scientists to unlock - has, over time, refined and developed the original proposal for a SKA. The outcome of this consultation process has been that the SKA aims to solve some of the biggest

* g.tan@skatelescope.org; phone +44 (0)161 306 9600; www.skatelescope.org

Ground-based and Airborne Telescopes VI, edited by Helen J. Hall, Roberto Gilmozzi, Heather K. Marshall, Proc. of SPIE Vol. 9906, 990660 - (c) 2016 SPIE · CCC code: 0277-786X/16/\$18 · doi: 10.1117/12.2230897 
questions in the field of astronomy. Eventually in phase SKA2, the unprecedented receiving sensitivity of the thousands of individual radio receivers, combining to create the world's largest radio telescope will give astronomers insight into the formation and evolution of the first stars and galaxies after the Big Bang, the role of cosmic magnetism, the nature of gravity, and possibly even life beyond Earth.

A complete, very detailed overview of the astronomical science is covered in the SKA documents ${ }^{2,3}$. Based on the science drivers a set of highest level functional and performance requirements for the SKA radio telescopes has been extracted and formalized ${ }^{4}$.

\subsection{SKA Observatory}

Figure 1 shows the major SKA Observatory entities: SKA1-Low in Australia (AU), SKA1-Mid in South Africa (SA) and the SKA Global Headquarters in the United Kingdom (UK). The thick flow-lines show the uni-directional transport of large amounts of digitised data from the receptors to the Central Signal Processing (CSP) facilities on the sites, and from the CSP facilities to the Science Data Processing Centres and Archives. The thin dash-dot lines show the bi-directional transport of system monitor and control data.

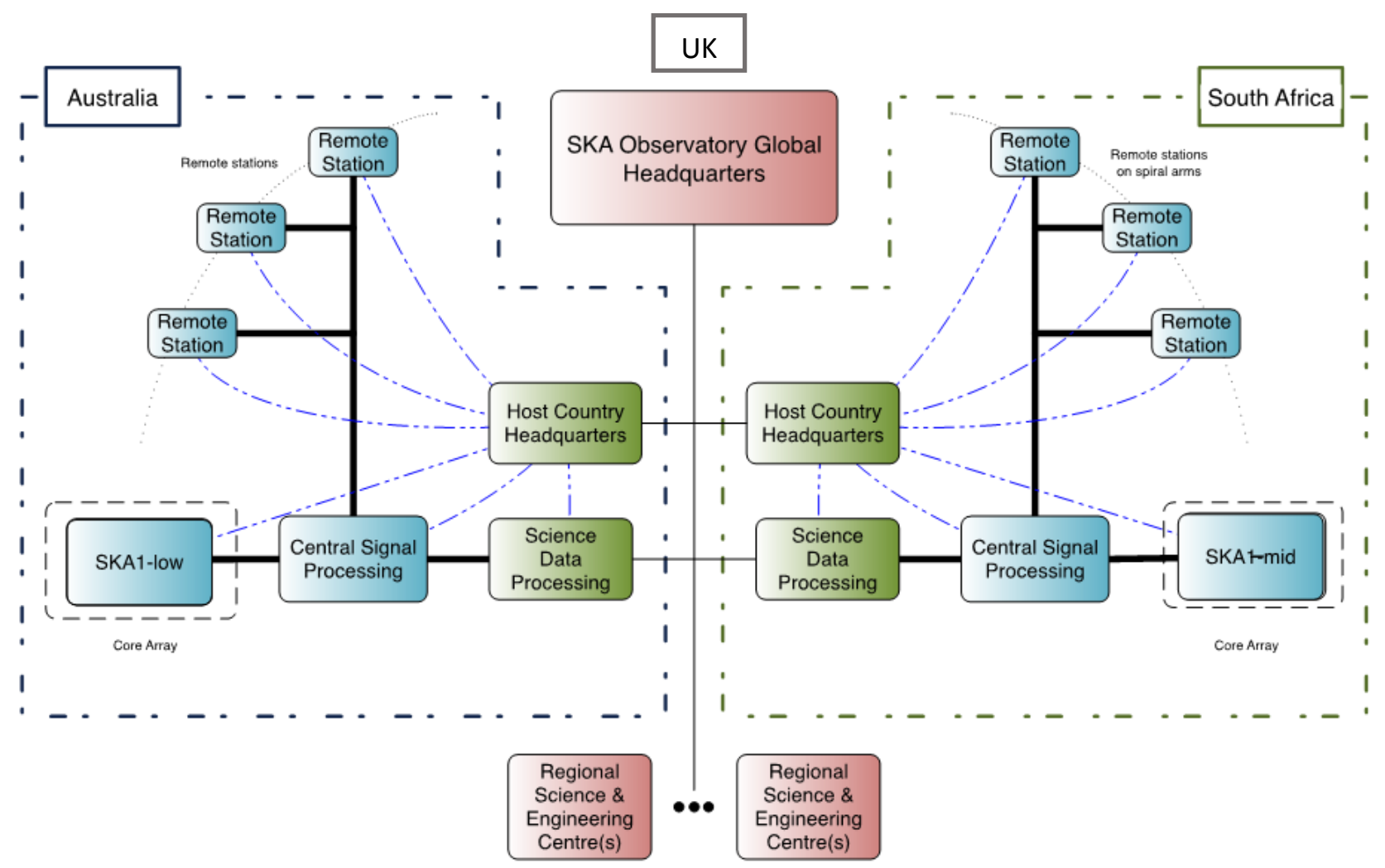

Figure 1. A schematic diagram of the SKA Observatory, showing the geographical locations of site entities (telescopes), the entities at regional centres (Host Country Headquarters and Science Data Processing), and entities that are globally located (Global Headquarters and RSEC/RDCs).

The Science Data Processor is envisaged to be a supercomputing facility with an attached or nearby archive to store science-ready data. The science data processor is where calibration of the data takes place, images of sky brightness are formed, and further analysis of time-domain observations are carried out. For current aperture synthesis arrays, algorithms for carrying out calibration and imaging are mature at higher frequencies. However, the SKA1-Mid is likely to require significant new developments in this area to handle the much larger amount of data, and to achieve the ambitious dynamic range targets without continuous human input. 
The Archives will store the outputs from the Science Data Processors in the site countries, where they will be kept for an indefinite time. The Regional Science and Engineering Centres (RSEC) and Regional Data Centres (RDC) are the facilities where it is expected that actual science analysis will take place and/or science data will be stored, as well as engineering design work for upgrades and future developments might take place. Apart from very general descriptions, their number and precise scope is undefined at this point and are place-holders for functions still to be defined.

\section{SKA1_MID SYSTEM DESIGN CONCEPT}

The SKA1-Mid telescope will be a mixed array of $13315 \mathrm{~m}$ SKA1 dishes and $6413.5 \mathrm{~m}$ diameter dishes from the MeerKAT telescope ${ }^{6}$. The antennas will be arranged in a moderately compact core with a diameter of $\sim 1 \mathrm{~km}$, a further 2dimensional array of randomly placed dishes out to $\sim 3 \mathrm{~km}$ radius, thinning at the edges (Figure 2). Three spiral arms will extend to a radius of $\sim 80 \mathrm{~km}$ from the centre (Figure 3).

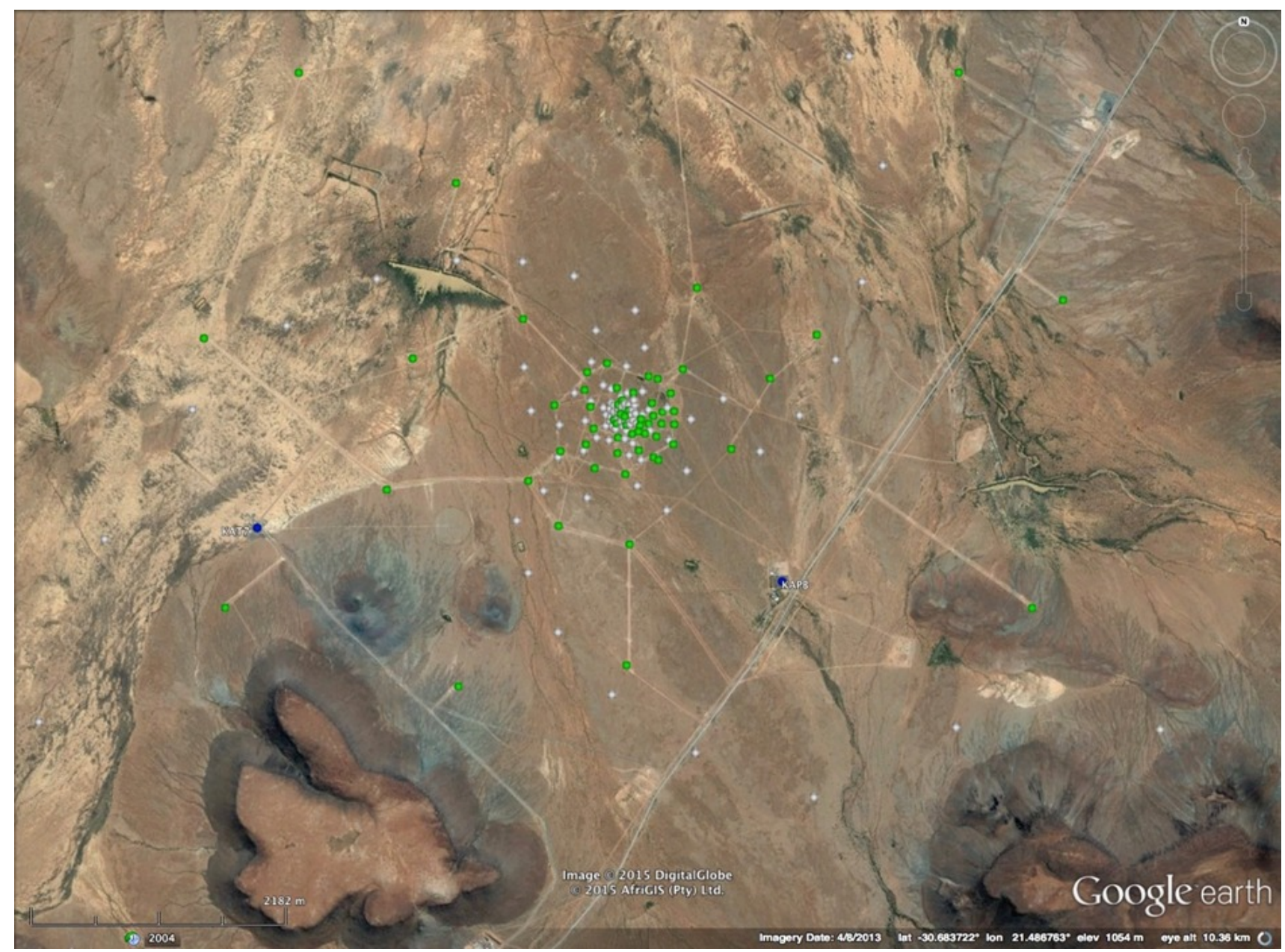

Figure 2. Close-up of SKA1-Mid core (white) on the Karoo Radio Astronomy Reserve, along with MeerKAT antennas (green) and various facilities (dark blue). 


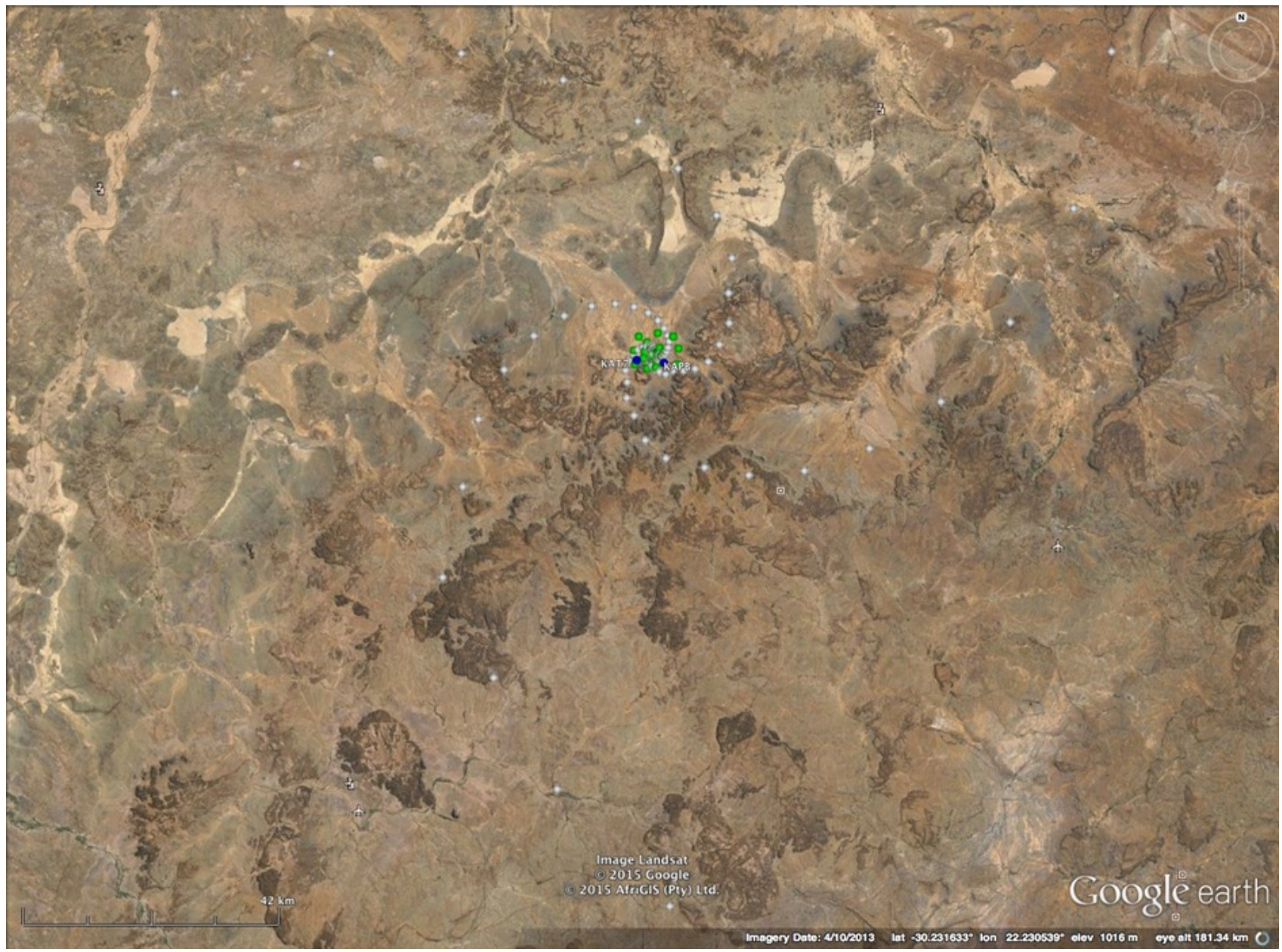

Figure 3. Overview SKA1-Mid antennas (white) on the Karoo Radio Astronomy Reserve, along with MeerKAT antennas (green) and various facilities (dark blue).

The dishes (Figure 4) will be clear-aperture, offset Gregorian optics design, capable of handling five low-noise front-end packages, each of which can be moved into the focal position. The antenna primary reflector surface is composed of panels. Except for the lowest receiver band (Band 1), the Single Pixel Feeds' (SPF) front-end, will be cryo-cooled. The dishes will be capable of operations up to at least $20 \mathrm{GHz}$, although initially equipped to observe only up to $13.8 \mathrm{GHz}$ for SKA1 distributed over five RF bands:

$$
\begin{aligned}
& \text { Band 1: } 350-1050 \mathrm{MHz} \\
& \text { Band 2: } 950-1760 \mathrm{MHz} \\
& \text { Band 3: } 1650-3050 \mathrm{MHz} \\
& \text { Band 4: } 2800-5180 \mathrm{MHz} \\
& \text { Band 5: } 4600-13800 \mathrm{MHz}
\end{aligned}
$$

Based on the science objectives the priority of these five receiver bands in descending order is Band 2, Band 5, Band 1, Band 4, and Band 3. Due to budgetary constraints SKA1-Mid will be equipped at the start of operations with Bands 1, 2 and 5. Efforts are underway to secure funding to equip the array with Bands 3 and 4 during SKA1-Mid operations.

After amplification of the RF signal it is directly digitized near the SPF package using a very precise, common sampler clock provided by a central frequency standard using an ensemble of H-masers. After digitization each of the data samples is time-stamped at the antenna avoiding susceptibility to delay variations in the data transport between antenna and back-end. 


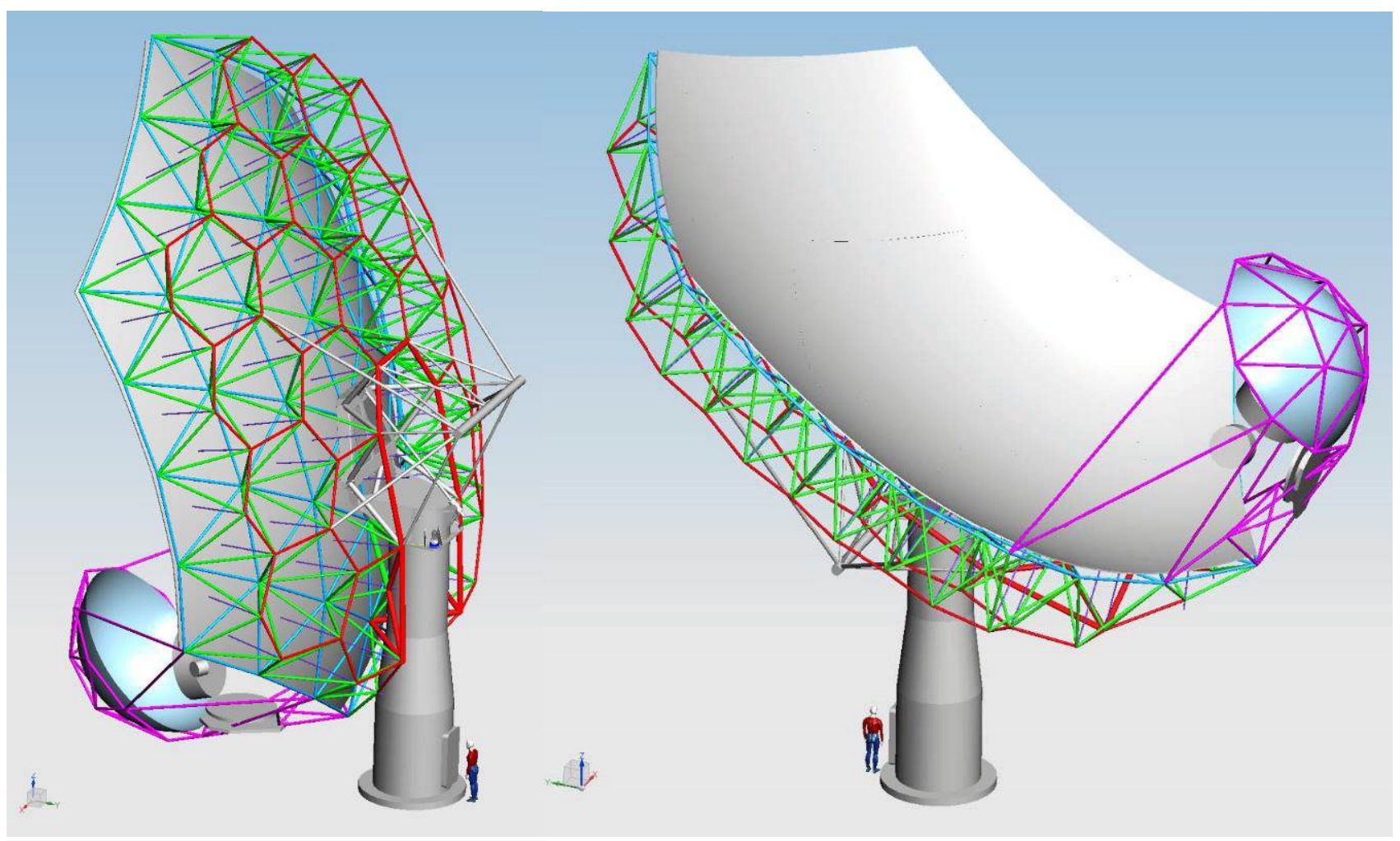

Figure 4. SKA1-Mid dish design: left rear view, right front view.

Signals from the dishes will be transported by optical fibre using a $100 \mathrm{Gbit} / \mathrm{s}$ Ethernet protocol to a Central Signal Processing Facility, where they will be divided into narrow frequency channels and cross-correlated with each other. Output data from the correlator will be transported to the Science Data Processing Centre in Cape Town.

The signals from the dishes will also be combined into a large number of array beams, the outputs of which will then be distributed to specialised pulsar search equipment. Pulsar candidates will be sent to the Science Data Processing Centre for further analysis. This equipment will also have some capability for detecting de-dispersed transients (rare or one-off, potentially extra-terrestrial radio-burst signals).

As for the other telescopes, the required processing of the science data will be varied, probably elaborate, and will likely include calibration, image-cube (i.e., spatial plus spectral) formation on various scales, time-domain analysis and statistical analysis.

\section{ANTENNA OPTICAL DESIGN}

Offset Gregorian reflector topologies (as opposed to symmetrical and prime focus fed paraboloidal systems) are the preferred choice for the optics of modern radio telescopes due to the absence of the feeds and struts blockage, and strut scattering ${ }^{7}$. Blockage decreases the effective aperture area and causes gain ripple with frequency. Strut scattering increases far-out sidelobe levels (SLLs) making the system susceptible to ground-based and satellite RFI. A further advantage of a dual reflector topology is that the reflectors can be shaped to improve the secondary aperture distribution, in other words the beam pattern, resulting in increased aperture efficiency and possibly improved SLLs.

In general, a classical conic section (unshaped) offset Gregorian reflector system is described by 5 parameters if the Mizugutch condition for optimal cross-polarisation is satisfied. One of these parameters is the aperture diameter, which 
is dependent on the telescope's collecting area, number of dishes and cost. It was chosen as $15 \mathrm{~m}$ for SKA1-Mid. Detailed parametric studies considering the remaining 4 parameters were performed ${ }^{8}$. Initially both ideal Gaussian feeds and realisable feeds optimised for particular reflector systems were investigated ${ }^{9}$. The two dominant parameters effecting the radiation performance are the sub-reflector size, which determines the performance at the lower frequencies, and the feed subtended half-angle $\theta_{e}$. Some of the parameters have a direct impact on cost and the final choice of the reflector system is a trade-off between cost and performance.

The most important metric for a radio telescope is its receiving sensitivity, which is proportional to the ratio of the aperture efficiency and system noise temperature. The sensitivity can be optimised for a given SLL by shaping the reflector system. Maximising the aperture efficiency does not, in general, correspond to maximising the receiving sensitivity. Note that MeerKAT, a precursor for the SKA1-Mid, had slightly different specifications than SKA1-Mid and thus used unshaped dishes due to lower required sidelobe levels.

\subsection{Tipping and sub-reflector extension}

The ground radiates thermal noise and any (spillover) illumination thereof will add to the system temperature, reducing the system sensitivity ${ }^{10}$. An additional parameter considered in the dish design was oversizing the sub-reflector at the bottom edge as shown in Figure 5. The size of this extension is defined by an angle which is measured from the reflector system's focus. This extension in combination with feed-down tipping (the reflector system is tilted by an angle from zenith in the plane of symmetry towards the horizon in the direction of the sub-reflector) reduces the noise contribution from the ground and allows increased illumination efficiency. This is demonstrated in Figure 6 for an ideal Gaussian feed with varying edge taper on an unshaped offset Gregorian dish. Note the higher sensitivity and flatness over a wide tipping angle for the feed-down tipping case. Feed-down tipping has the added logistical advantage that the feeds are accessible closer to ground level.

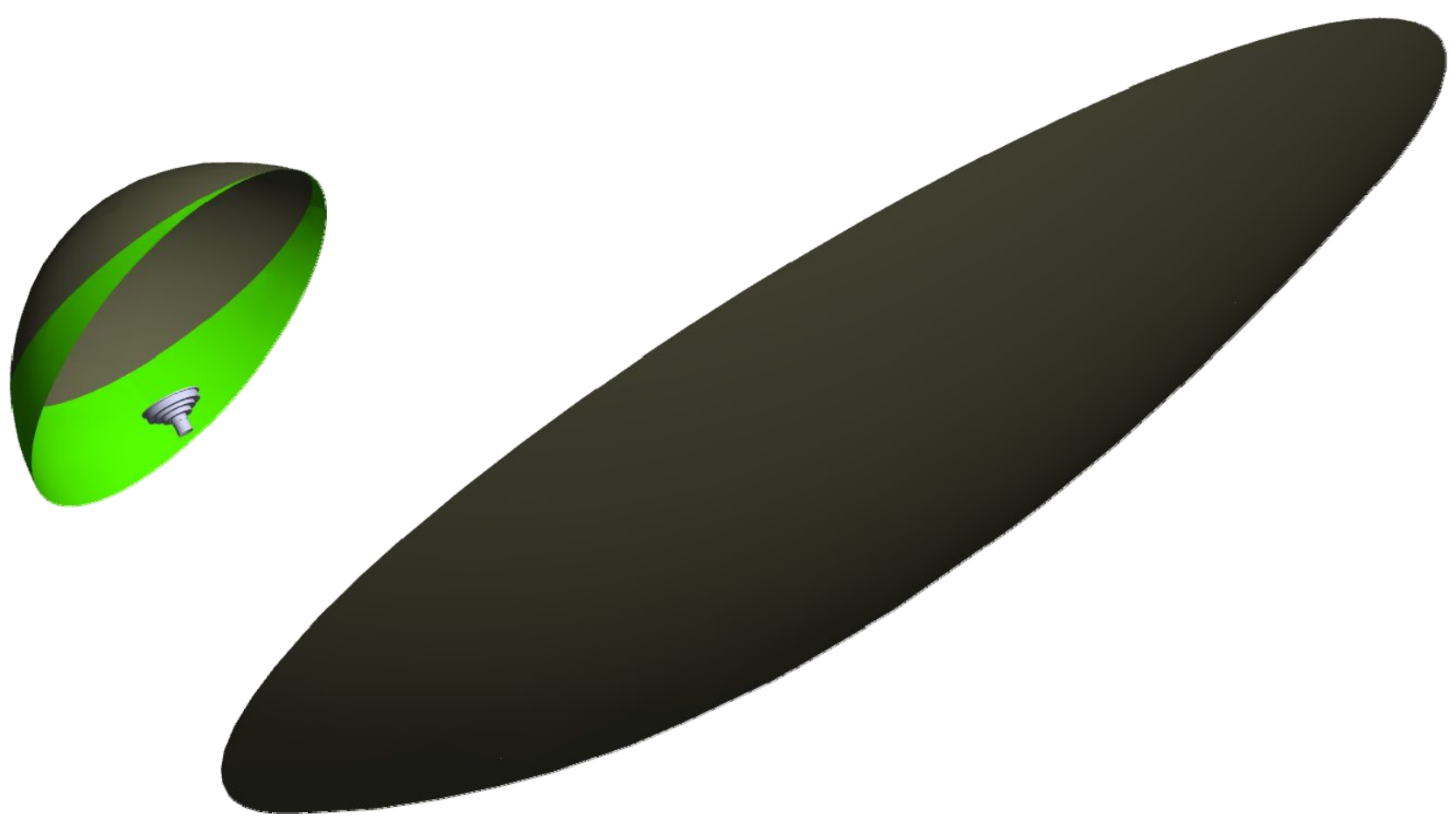

Figure 5. A model of the offset Gregorian reflector system with the green section highlighting a $40^{\circ}$ sub-reflector extension. 

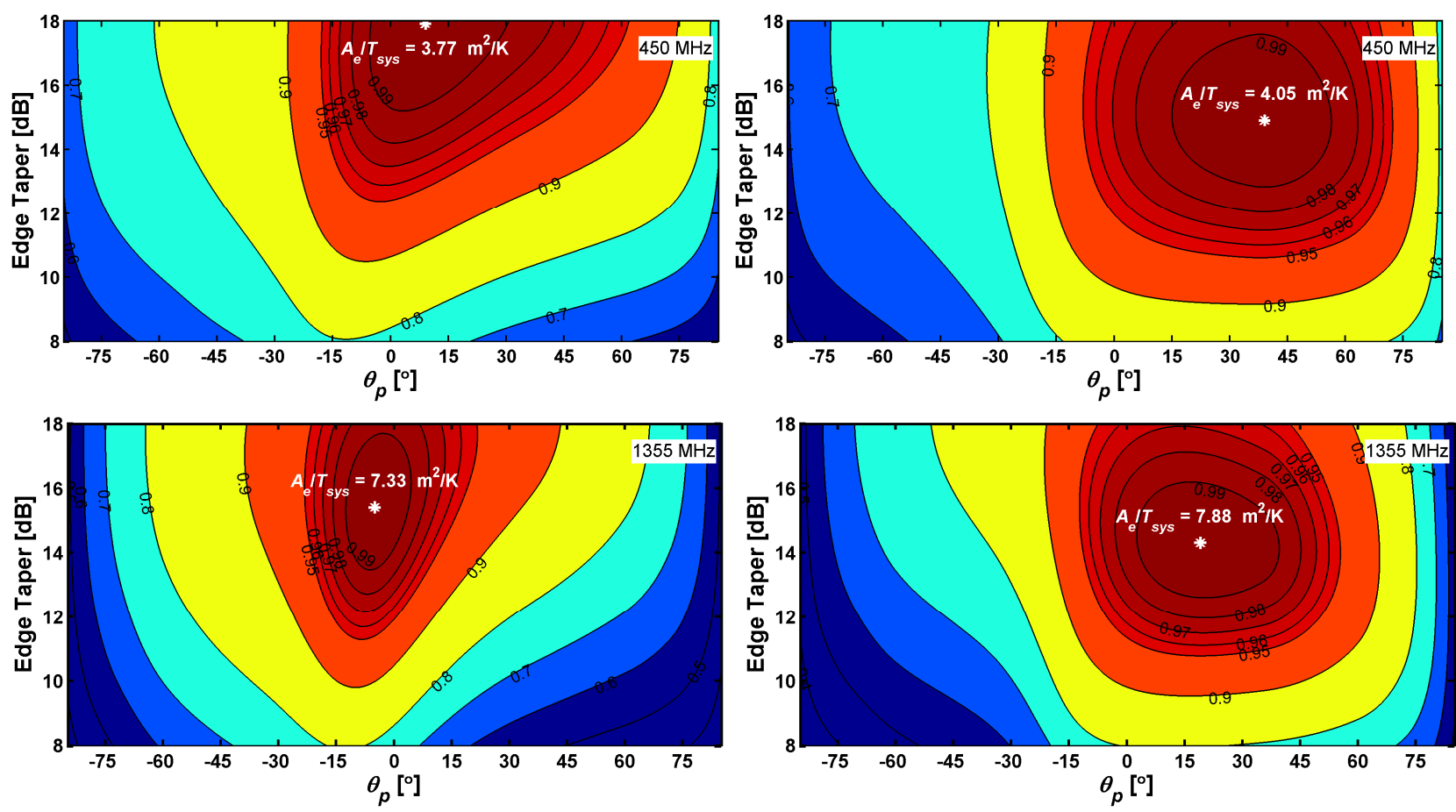

Figure 6. Normalised receiving sensitivity of a Gaussian beam with varying edge taper illuminating an unshaped offset Gregorian dish with an aperture diameter of $15 \mathrm{~m}$ without (left) and with (right) a sub-reflector extension of $20^{\circ}$ and assuming a receiver noise temperature of $10 \mathrm{~K}$. Feed-down tipping is defined with $\theta_{p}$ positive.

\subsection{Shaping optimisation}

Shaping the reflector system improves sensitivity over an unshaped system; firstly, the illumination efficiency is improved at the cost of increased near-in SLLs, and secondly the spillover is reduced by moving the feeds closer to the sub-reflector and reducing the edge illumination.

The shaping procedure needs to establish a mapping function that transforms a given feed pattern to an aperture field distribution with a desired secondary (reflector system) radiation pattern. Given the mapping function the reflector surfaces can be determined using geometric optics. The frequency bands for SKA1-Mid have different relative bandwidths and thus require different feed technologies, which then have different feed pattern responses over frequency. Any variation over frequency of the feed's radiation pattern will cause changes in the secondary radiation pattern. Furthermore, the secondary pattern will also vary over frequency due to effects of diffraction from especially the sub-reflector edge. Thus, the design of a mapping function that is suitable over the entire SKA1-Mid frequency band, according to some performance metric, is a difficult optimisation problem.

The procedure followed was as follows ${ }^{11}$. The mapping function was parameterised by assuming uniform/Gaussian functions for both the feed pattern and aperture field distribution. Then an interpolant was determined for the optimisation parameter space based on an equi-spaced grid of evaluation points. Given this interpolant the entire optimisation domain was searched exhaustively to establish the Pareto front of maximum average (over frequency and tipping angles) sensitivity for a given average (over frequency) second sidelobe level $\left(\mathrm{SLL}_{2}\right)$ - the first SLL is accounted for in the astronomy calibration and the near-in sidelobe levels are typically decreasing from the main beam. The optimisation was done using realistic receiver temperatures and frequency-dependent feed radiation patterns as determined from feeds optimised for geometrically similar unshaped reflector systems. Accurate calculation of the sensitivity is computationally expensive due to the antenna noise temperature calculation. This entails integrating the product of the secondary radiation pattern with the surrounding scene brightness temperature ${ }^{11}$ over the entire $4 \pi$ steradian sphere, and this varies as a function of frequency and tipping angle. In order to make the shaping optimisation tractable a number of approximations had to be made and have been documented ${ }^{12}$. 
The main objective was to design a shaped system that improves the performance of the Band 1 feed without seriously degrading the performance of the Band 2 and higher frequency band feeds. The sensitivity of the wider band Band 1 feed is generally lower than the rest. Although not discussed in this paper, cluster type feed systems that generate multiple beams were also considered in the reflector shape, as they may be considered for future upgrades ${ }^{13}$.

Figure 7 summarises the outcome of the shaping optimisation. Shown are the Pareto fronts for various sub-reflector sizes and feed subtended angles. It is observed that the sub-reflector size only marginally improves the performance. A shaped system that minimizes the edge illumination has the best performance. To prevent the feed from entering the optical path of the reflector system $\theta_{e}$ cannot be increased beyond $58^{\circ}$. Optimising for Band 1 does not significantly compromise the performance in Band 2. The final reflector system was chosen with a $-24 \mathrm{~dB} \mathrm{SLL}_{2}$ in Band 1 (or -26 dB SLL2 in Band 2). The additional requirement of reflectors to accommodate cluster type feeds push towards a $5 \mathrm{~m}$ sub-reflector.
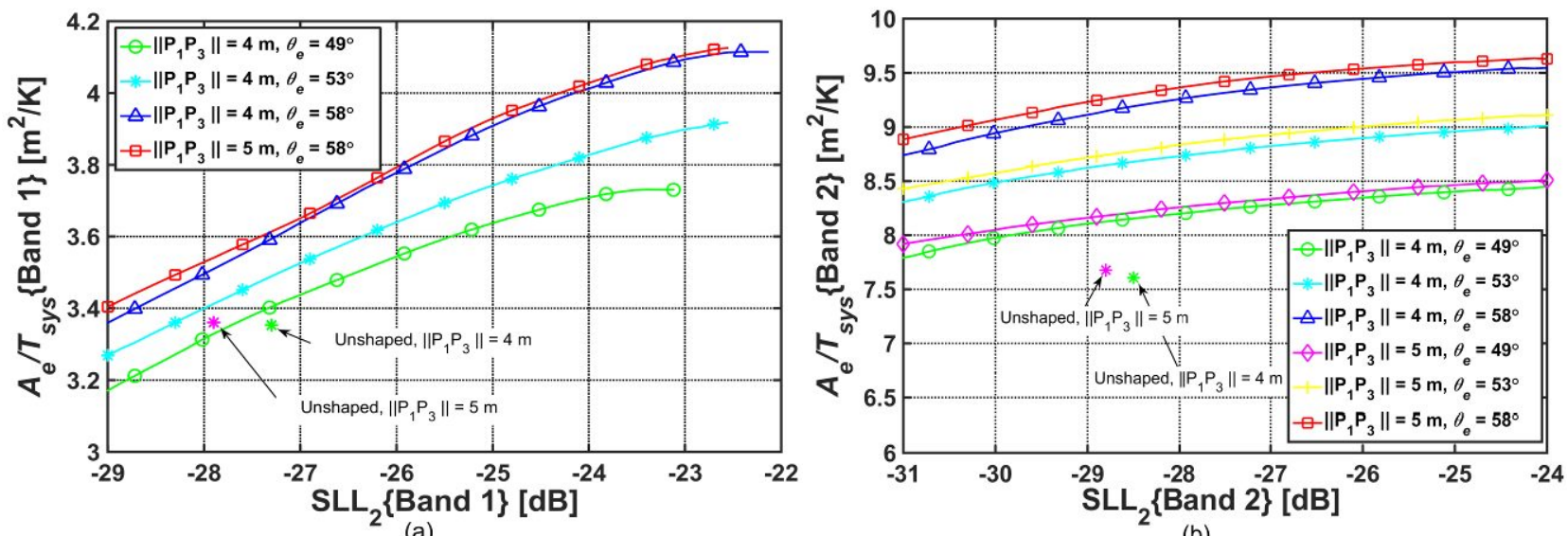

(b)

Figure 7. The maximum average receiving sensitivity for a given average $\mathrm{SLL}_{2}$ in (a) Band 1 and (b) Band 2 for dishes with sub-reflectors of $4 \mathrm{~m}$ and $5 \mathrm{~m}$ size and a $20^{\circ}$ extension, and various $\theta_{e}$ angles. For comparison the results for unshaped systems with $\theta_{e}=49^{\circ}$ are also shown.

\subsection{Sub-reflector extension optimisation}

The effect of increasing the size of the extension is investigated in this section. The sub-reflector size is kept at $5 \mathrm{~m}$ and the extension is increased to $50^{\circ}$. Again the shaping optimisation is repeated for each case. The results are given in Figure 8 .
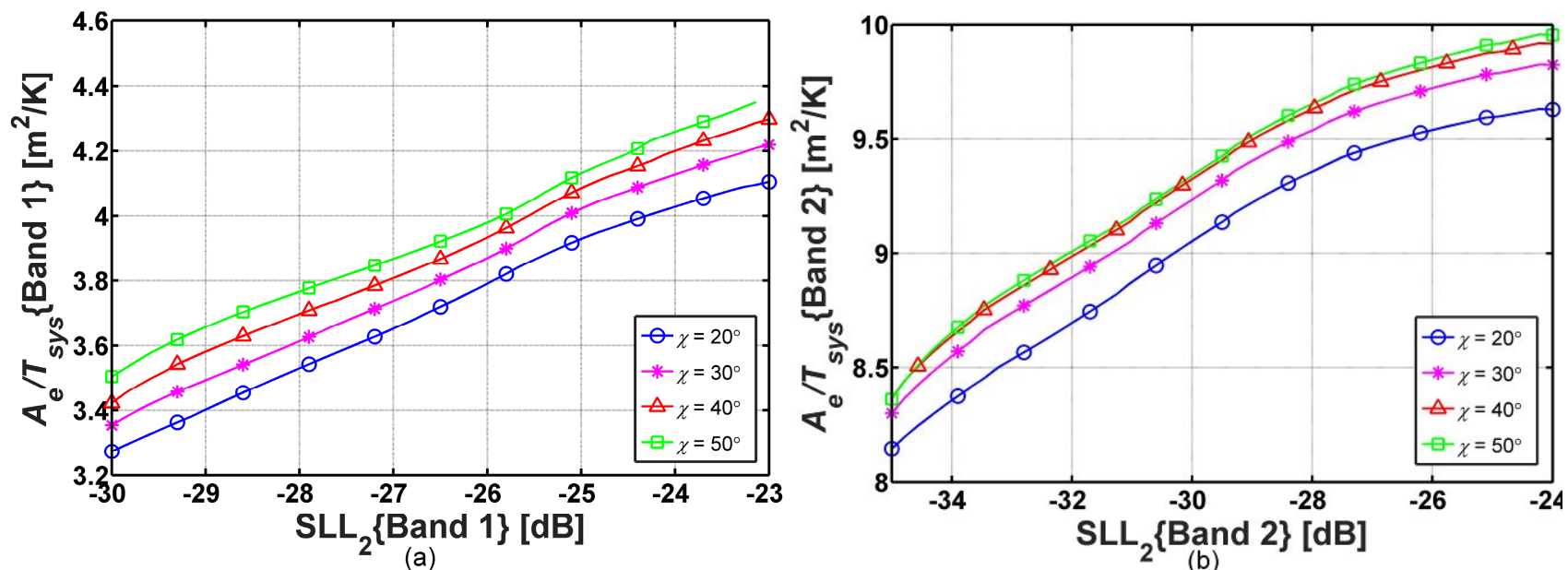

Figure 8. The maximum average receiving sensitivity for a given average $\mathrm{SLL}_{2}$ in (a) Band 1 and (b) Band 2 for dishes with $\theta_{e}=58^{\circ}, 5 \mathrm{~m}$ sub-reflectors with various extension angles, $\chi$. 
For a larger extension the spillover temperature decreases and the receiving sensitivity increases. The size of the extension is, of course, limited by geometrical constraints. In Band 2 the advantage of the added extension is maximized for an angle of $40^{\circ}$. A flattening off is also seen in Band 1. The final selected optics has a feed angle of $58^{\circ}$ and a $5.16 \mathrm{~m}$ sub-reflector which includes a $40^{\circ}$ bottom extension.

\section{SINGLE PIXEL FEED RECEIVER DESIGNS}

The SKA1-Mid telescope will initially have only Bands 1,2 and 5 installed due to budget constraints. Band 5 is a recent addition following a SKA Project re-baselining, including a science reprioritization, in 2015 and not covered in this article.

\subsection{SKA1-Mid Band 1}

It was originally envisioned that the SKA Band 1 Feed Package would use a Quad-Ridged Flared Horn (QRFH) at ambient temperature and cryogenically cooled LNAs. The performance of the QRFH in the different optics designs considered for SKA are described in the optics analysis report ${ }^{[14]}$. The QRFH was selected after comparing cost and performance aspects with the Eleven Feed, the other feed considered as possible candidate for Band $1^{[15]}$.

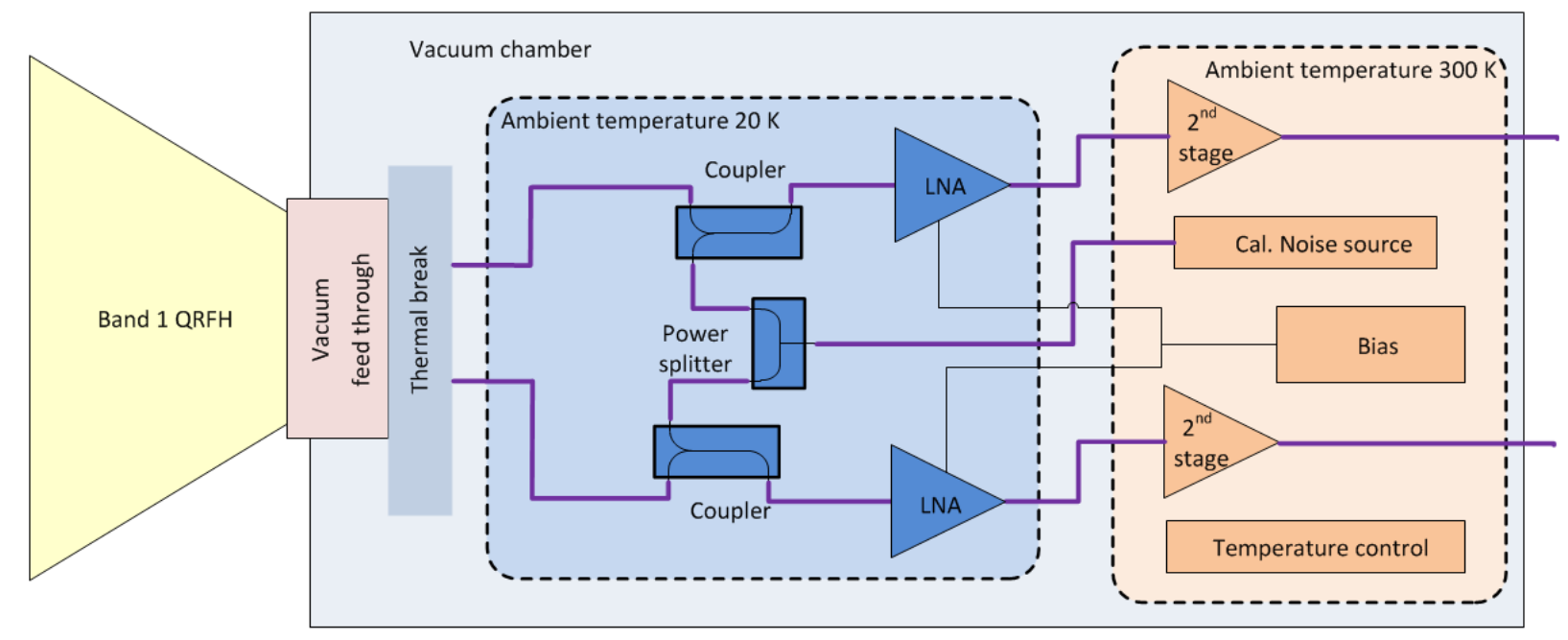

Figure 9. Overview of Band 1 feed system.

Figure 9 shows the design concept for the Band 1 feed system. The QRFH is at room temperature and attached mechanically to the front plate of the cryostat. The RF interface to the signal chain is provided by vacuum feed through connectors at the input of the cryostat. The heat transfer from $300 \mathrm{~K}$ to $20 \mathrm{~K}$ cold stage is minimised using stainless steel coaxial cables to transport the signal. These cables are anchored thermally at $20 \mathrm{~K}$ and $70 \mathrm{~K}$. The RF signal for each of the two orthogonal polarisations then passes through a directional coupler where calibration noise signal is injected, followed by a cryogenic LNA and 2nd stage LNA. The cryogenic LNAs and direction coupler are attached to the $20 \mathrm{~K}$ cold stage of the cryostat. The 2nd stage LNAs, and calibration noise source are located at the $300 \mathrm{~K}$ stage.

Figure 10 shows a cross section of the Band 1 cryostat, which uses a Gifford-McMahon (GM) cooler. The LNAs and the directional couplers are placed on thermally stabilized copper plate that is attached to the $20 \mathrm{~K}$ stage of the cryo-cooler using weak thermal links. A stainless steel (SS) co-axial cable is used between the vacuum feed through located at $300 \mathrm{~K}$ interface and the direction coupler at $20 \mathrm{~K}$, to reduce the thermal heat flow. Since the SS co-axial cable is located before the first stage LNA, loses of the SS cable add significant noise to the overall system noise, therefore length of this cable is carefully optimized to reduce the added noise contribution but at the same time providing enough thermal isolation between the two temperature stages. 


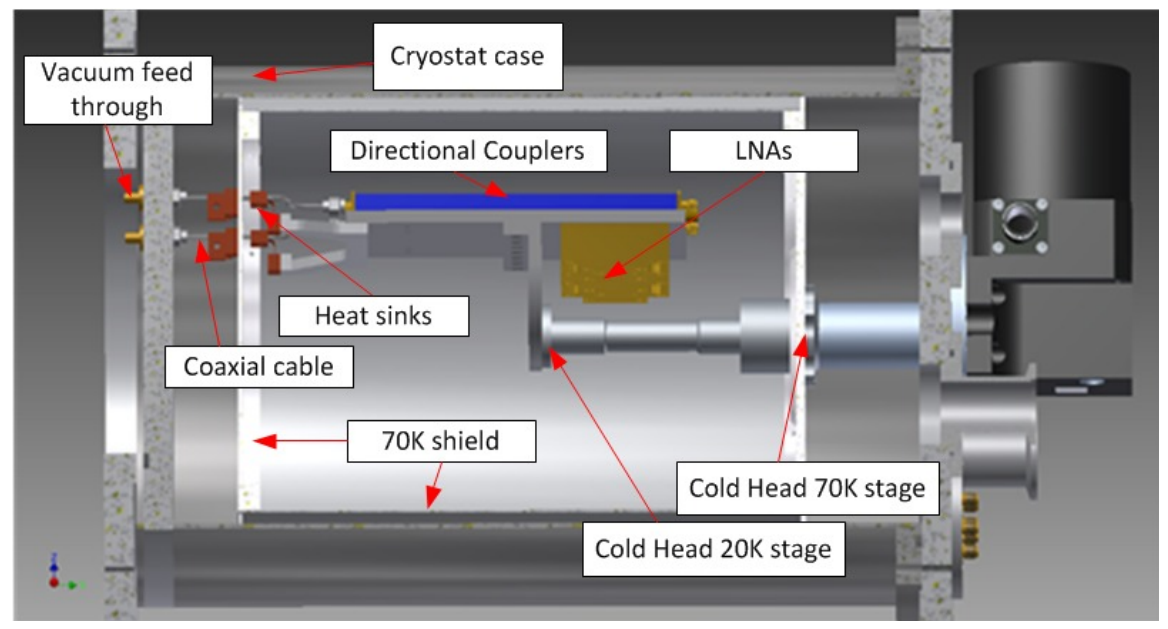

Figure 10. Overview of Band 1 cryostat.

The expected noise temperature of the cryostat at the co-axial input of the feed horn is shown in Figure 11. Where, $\mathrm{T}_{\text {Cryo }}$ is the noise temperature measured at the input of the cryostat which includes the cryogenic LNA, direction coupler and all the losses up to the $300 \mathrm{~K}$ interface of the cryostat. $\mathrm{T}_{\text {feed }}$ represents the estimated noise contribution from the feed at room temperature, and $\mathrm{T}_{\text {Backend }}$ is the noise contribution from all the subsequent components after the first cold LNA.

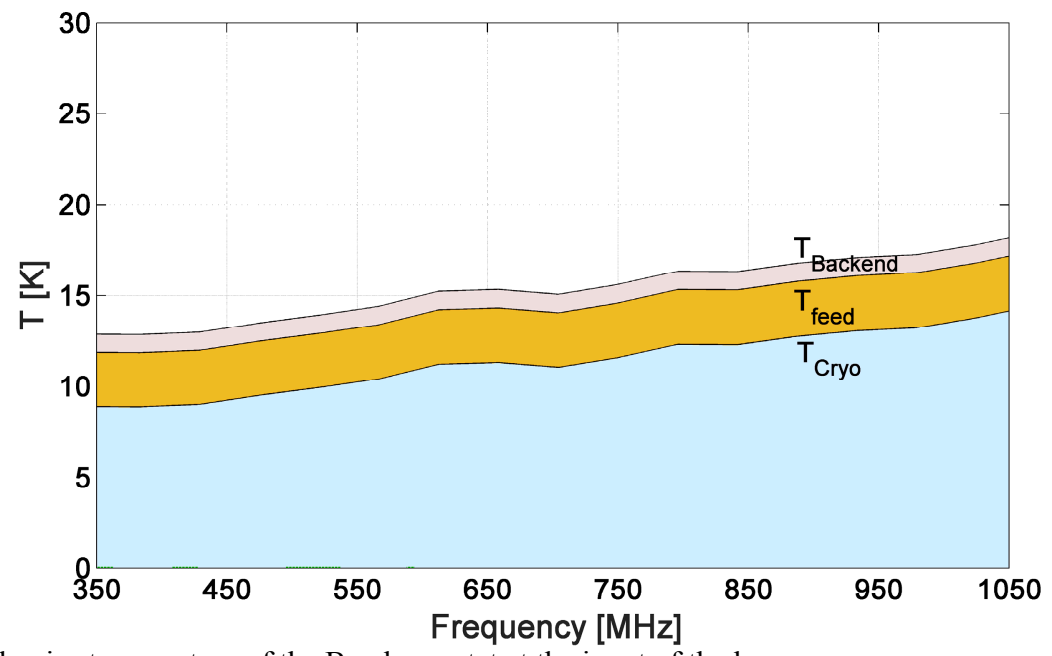

Figure 11. Measured noise temperature of the Band cryostat at the input of the horn.

The QRFH for the Band 1 of the SKA-mid has been designed to maximize the overall sensitivity of the telescope, by making trade-off between the aperture efficiency and spill over noise contribution over the entire band while keeping the feed input reflection coefficient in the acceptable range. Figure 12 a. shows the Band 1 QRFH, both the ridge profile, and outer horn profile is defined using spline function. In our simulations we observed that a spline profile horn provide better efficiency compared to exponentially tapered QRFH over 3:1 bandwidth. For wider bandwidths however, an exponential profile ${ }^{[16]}$ might still provide better efficiency. Figure $12 \mathrm{~b}$. shows the $\mathrm{CST}^{[17]}$ simulation results of reflection coefficient of the QRFH for both the ports. For both the ports the input reflection is better than $-10 \mathrm{~dB}$. The slight degradation in the input matching at the low end of the frequency band is believed to be associated with the ridge and horn profile, and not with the feeding section. By modifying the ridge and horn profiles the input reflection can be improved, but we observe reduction in the overall efficiency. However, we believe that further optimization of the feed could improve the input matching without substantially affecting the efficiency. 

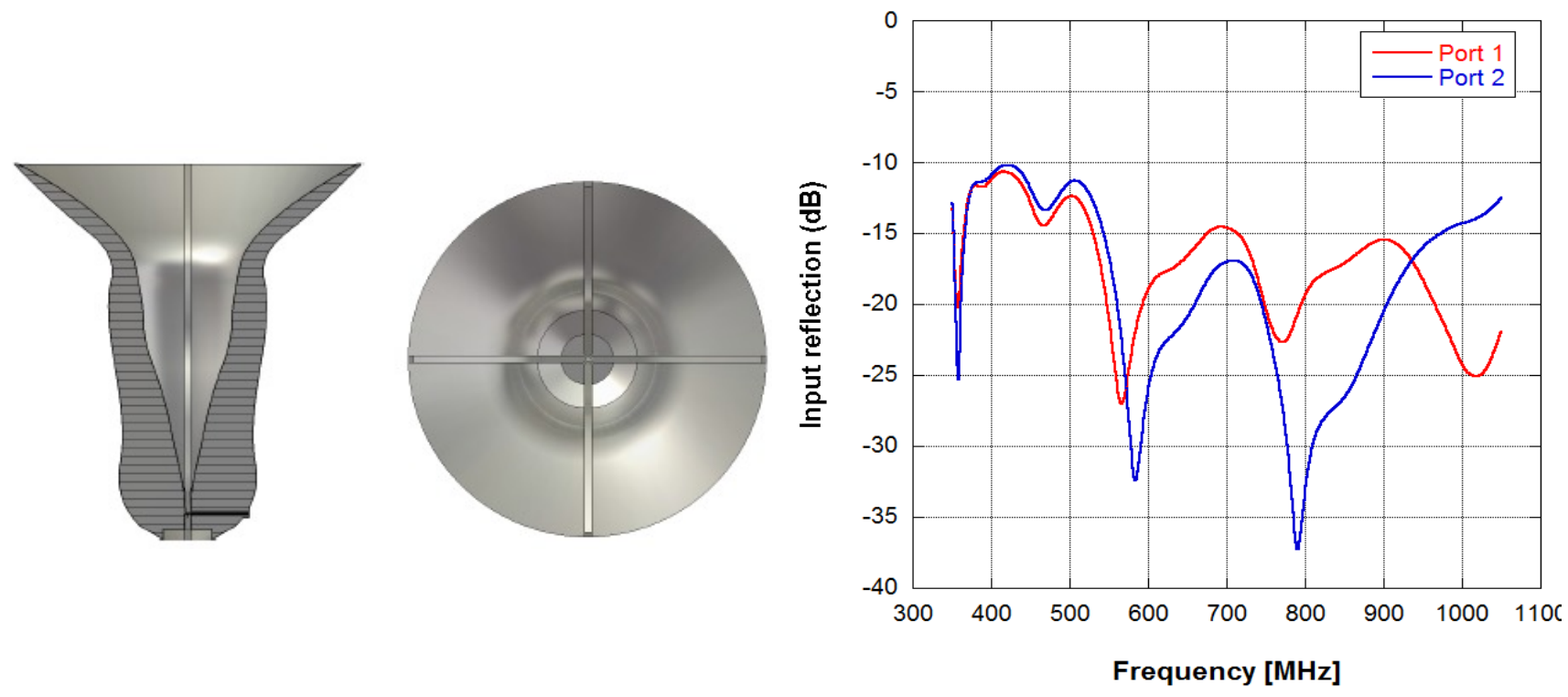

a.

b.

Figure 12. SKA Band 1 QRFH, a. CAD model of the feed horn, b. simulated reflection at the co-axial connector.

In order to accurately estimate the overall system sensitivity, one needs to accurately estimate the spill over noise contribution of the reflector system. The estimation of the overall on-sky sensitivity and optimization of the QRFH is done using a GRASP system simulator ${ }^{[18]}$, where the CST far field patters of the QRFH are analysed with the SKA dish geometry, as in Figure 5, using GRASP ${ }^{[19]}$. The simulated aperture efficiency of the QRFH in the SKA dual reflector geometry is shown in Figure 13 for both polarizations. The aperture efficiency is better than $75 \%$ over most of the frequency band and better than $70 \%$ over the entire band.

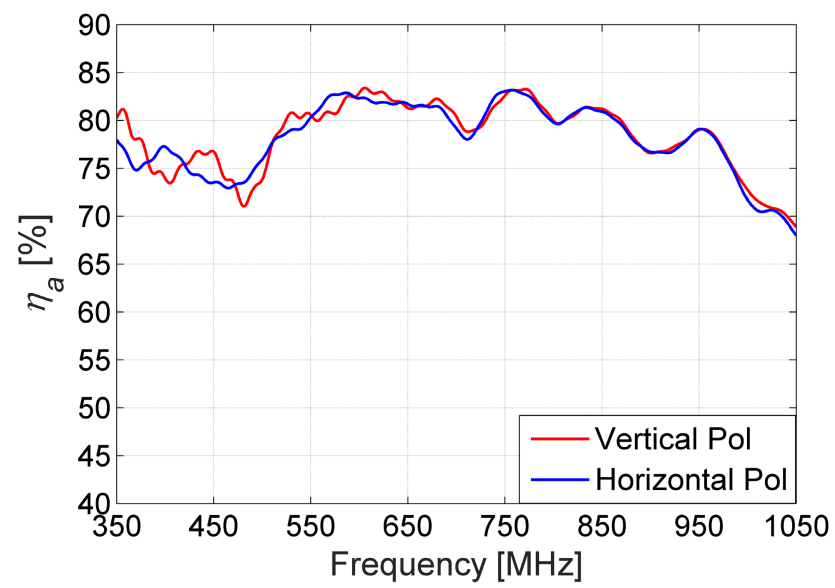

Figure 13. Simulated aperture efficienyt of Band 1 feed.

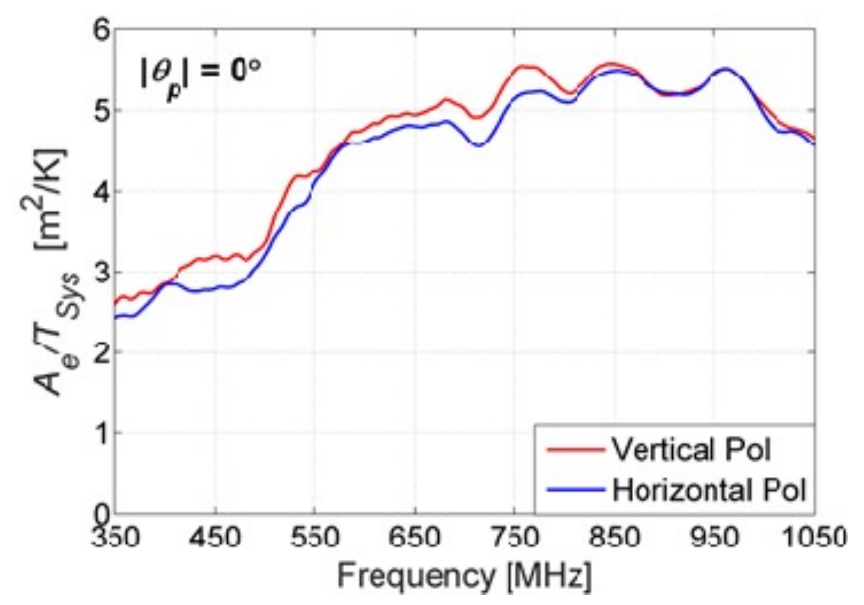

Figure 14. Simulated dish sensitivity $\left(\mathrm{A}_{\mathrm{eff}} / \mathrm{T}_{\text {sys }}\right)$ of the QRFH in the SKA.

Figure 14 shows the simulated system sensitivity of the feed in the SKA dish geometry, using the cryostat noise estimation described in previous section, and the analysis from GRASP and system simulator. The sensitivity is calculated when the dish is looking at zenith, for other elevation angles the overall sensitivity changes as a function of elevation angle. 
The estimated performance of Band 1 feed system indicate that even with high aperture efficiency of Band 1 feed, the overall performance of the feed system below $650 \mathrm{MHz}$ is limited due the sky noise. Secondly, one of the biggest noise contributions in the overall Band 1 cryostat noise described in Figure 10, are the passive losses associated between the feed and the cryogenic LNA.

Considering the high losses in the warm part of the baseline Band 1 system, we started investigating room temperature alternative with Low Noise Factory (LNF), Sweden. LNF made a custom design of a room temperature for SKA Band 1 with very promising performance ${ }^{[20]}$.

If the room temperature LNA is integrated directly on the ridges, minimizing the losses before the LNA the overall feed package noise would be then determined by the LNA noise only. Figure 15 shows preliminary estimate of the complete room temperature system, the performance of a complete room temperature Band 1 system is comparable with the cryogenic solution. A feed package using this room temperature LNA integrated in the ridge would have clear advantage over the cryogenic system in terms of capital cost, maintenance costs as well as reduced power consumption.

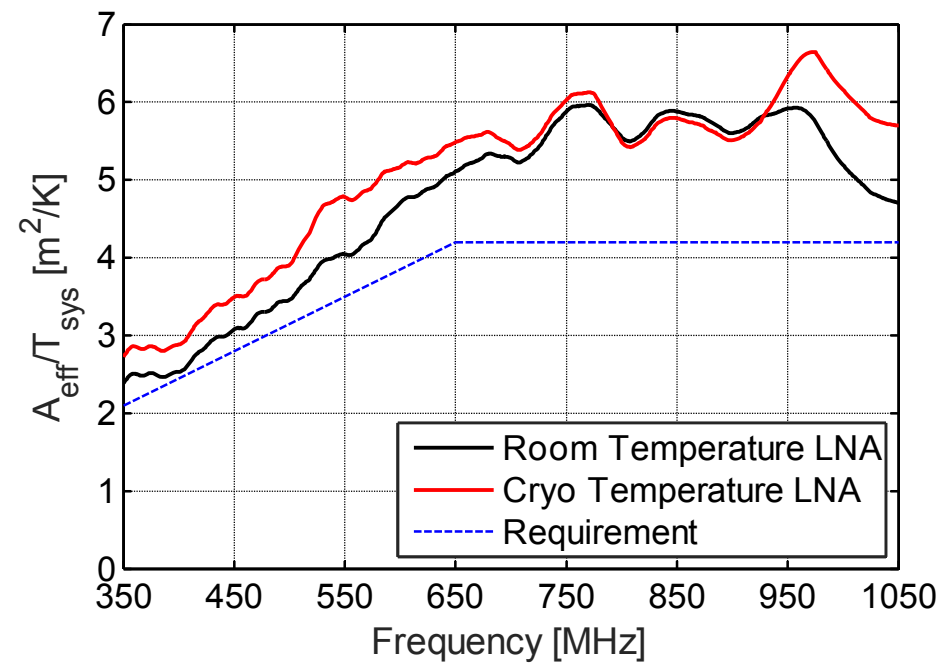

Figure 15. Expected performance of a complete room temperature Band 1 system and cryogenic system.

\subsection{SKA1-Mid Band 2}

The main components of the Band 2 feed are shown in Figure 16 and the topology is similar to the MeerKAT system ${ }^{21}$. It is a dual linearly polarized feed consisting of a wide flare angle axially corrugated conical horn and a compact orthogonal mode transducer (OMT) as illustrated in Figure 17. A thermal break - needed to allow cooling the lossy OMT to very low temperatures - is realized by a gap between the warm (room temperature) horn and circular waveguide feeding the horn, and the cold (100 K is assumed here as worse case) OMT's dipole and balun structure. The cryostat's barrier between vacuum and the atmosphere is realized by a high-density polyethylene dome.

This horn can be designed to have good pattern symmetry and cross-polar performance ${ }^{22,23}$. Two steps in the circular waveguide are used to match the $\mathrm{TE}_{11}$ circular waveguide mode feeding the horn to free space. The horn's parameters were determined with a hybrid optimisation algorithm that combined the Population-Based Incremental Learning (PBIL) global search algorithm with a downhill simplex method. The objective function consisted of a weighted sum of the following goals over the frequency band: maximum average receiving sensitivity, minimum $\mathrm{TE}_{11}$ mode reflection coefficient, limit the maximum sidelobe and cross-polarisation levels, and limit the drop-off of efficiency at the highest frequency. 


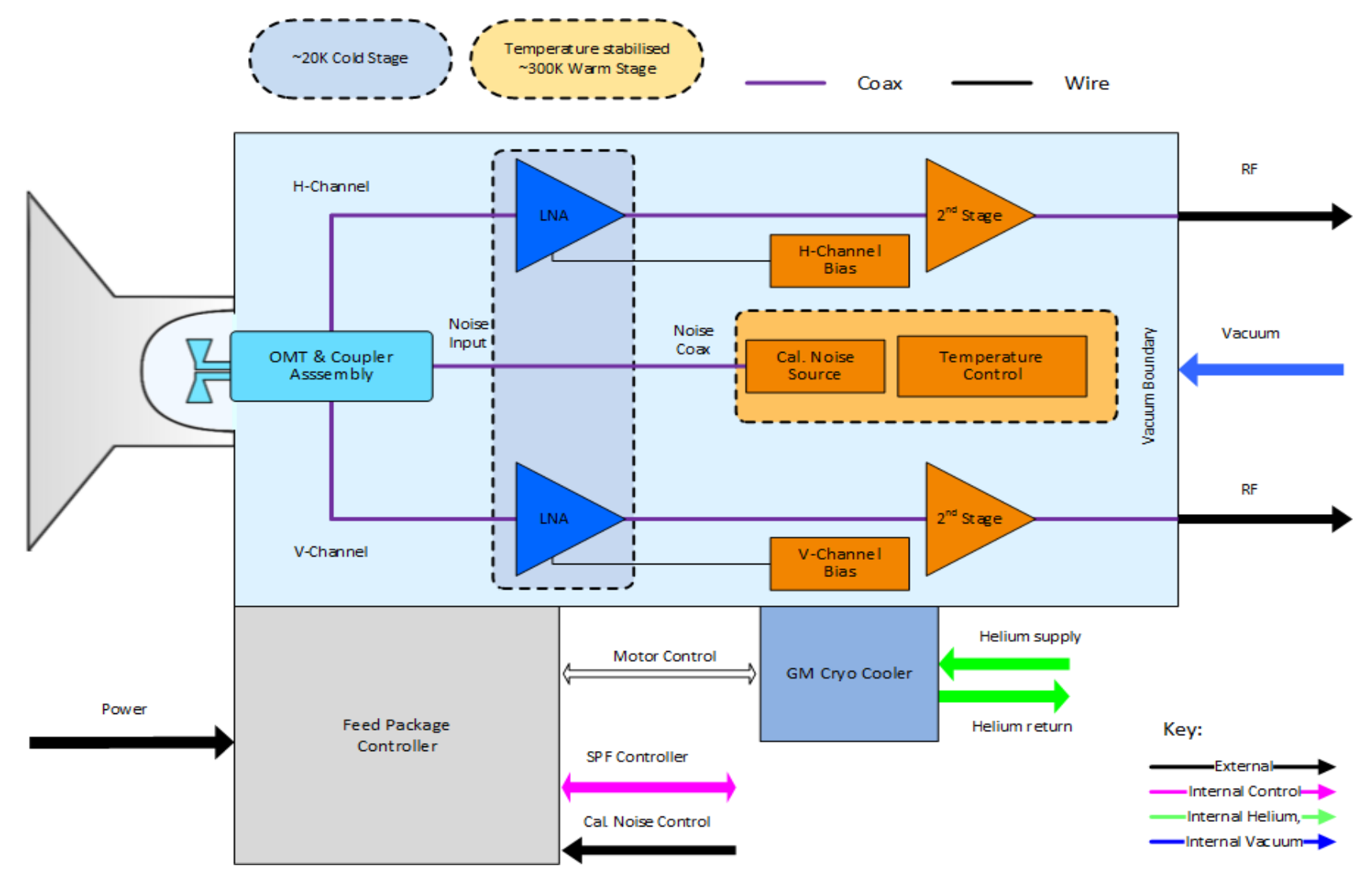

Figure 16. Diagram illustrating the main components of the Band 2 feed.

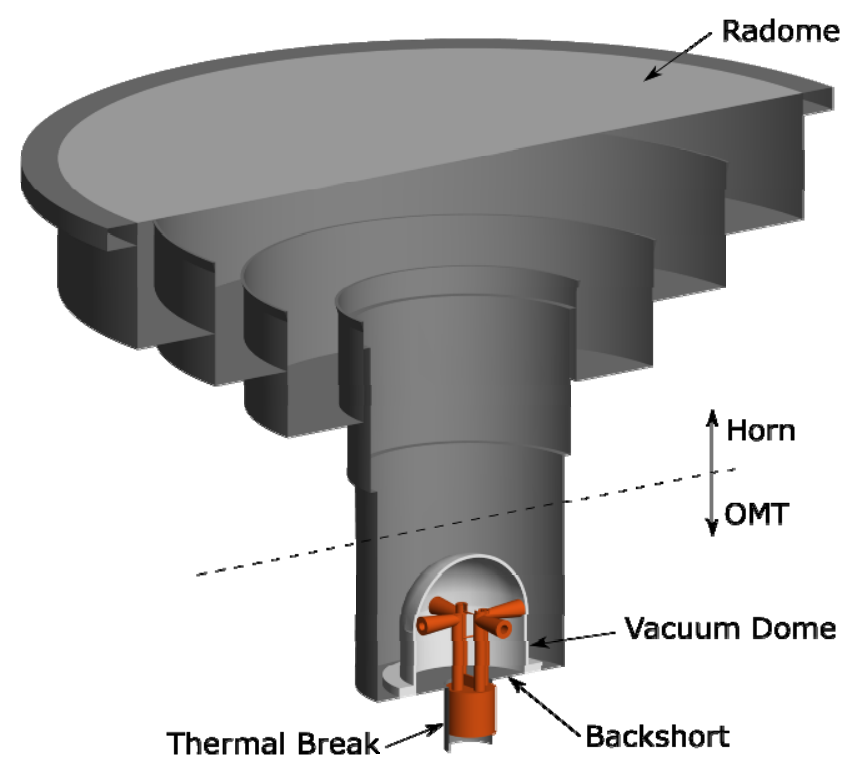

Figure 17. Model of the front-end of the Band 2 feed showing the horn, OMT, vacuum dome and thermal break. The wide flare angle axially symmetric corrugated conical horn includes the environmental radome, three corrugations, and two matching steps. The OMT consists of a pair of crossed-dipoles with integrated folded Marchand baluns and the thermal break which houses the calibration noise coupler. 
In each iteration of the optimiser the electromagnetic (EM) analysis followed a two-step process. Firstly, the horn was analysed using a method of moments solver, namely $\mathrm{FEKO}^{24}$, to determine the $\mathrm{TE}_{11}$ mode reflection coefficient into free space and the horn's radiation pattern. The effect of the sub-reflector on the horn's reflection coefficient is a ripple superimposed on the free space reflection coefficient. This effect cannot be minimised through feed optimisation. The feed's phase centre was determined from the horn's radiation pattern ${ }^{25}$. Secondly, the feed (using the spherical mode expansion coefficients), positioned at the focus, was used to illuminate the reflector system using the physical optics and physical theory of diffraction method of GRASP ${ }^{19}$ to determine the reflector's far field. In this optimisation an ideal OMT was assumed, i.e. using symmetry and feeding the horn with a $\mathrm{TE}_{11}$ mode.

After the full optimisation, the combination of the horn and dish were analysed in both GRASP and FEKO over the specified frequency band. The FEKO predicted horn and dish radiation patterns at the centre frequency are given in Figure 18 and Figure 19 respectively. The patterns are stable over frequency.

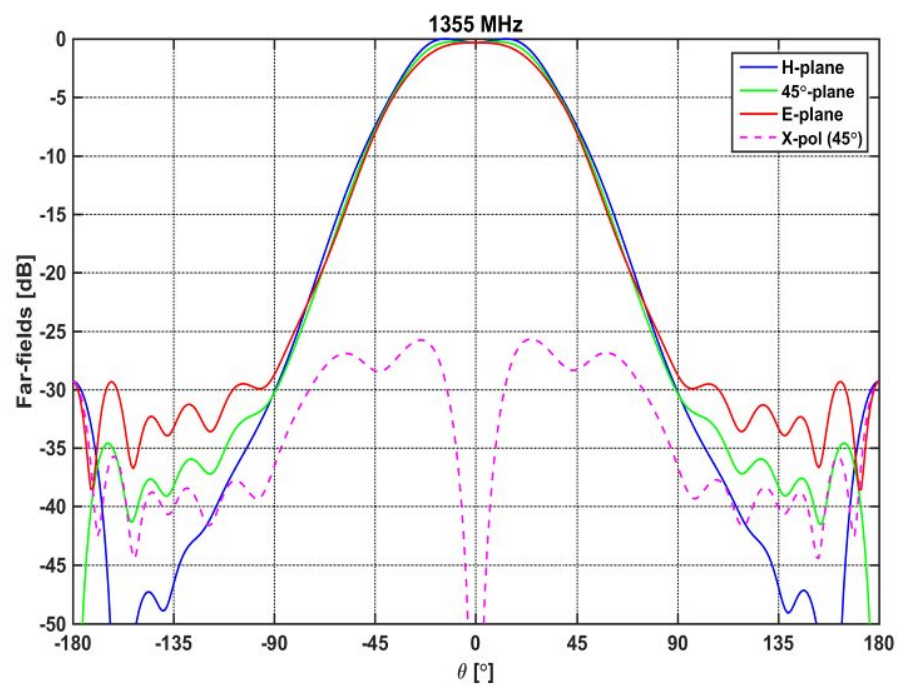

Figure 18. The optimised horn's radiation pattern at $1355 \mathrm{MHz}$.

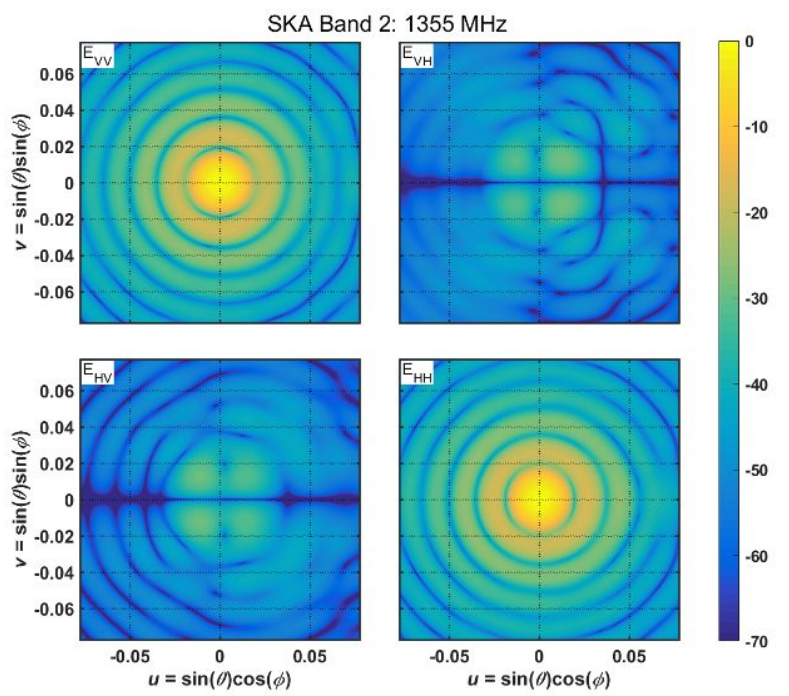

Figure 19. The projected co- and cross-polarised radiation patterns, i.e. Jones matrix, of the main beam and first few SLLs of the horn and reflector system at $1355 \mathrm{MHz}$.

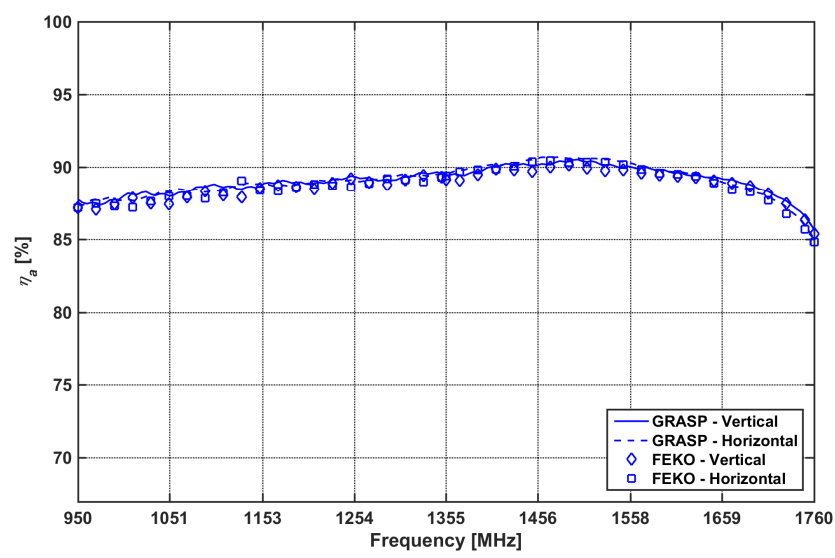

Figure 20. The aperture efficiency. Both GRASP and FEKO analyses are shown for both polarisations of the reflector system illuminated with the optimised horn.

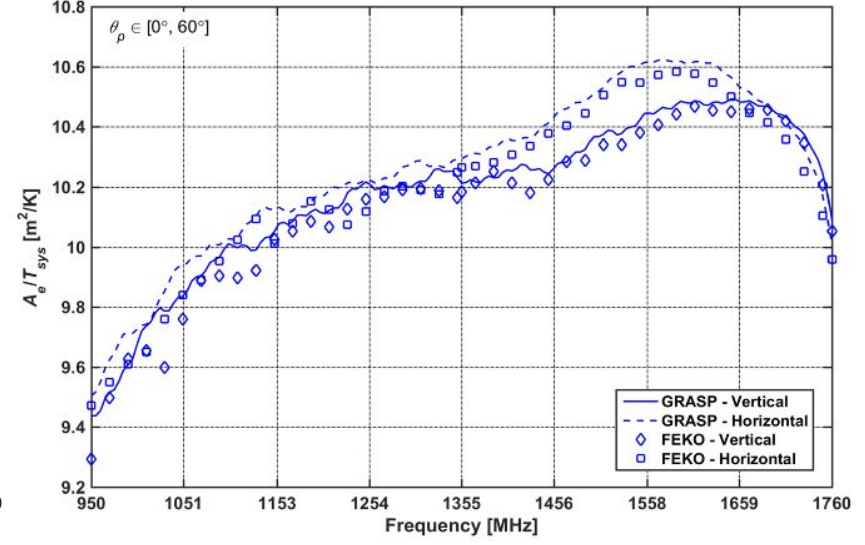

Figure 21 . The average receiving sensitivity over all pointing angles $\theta_{p} \in\left[0^{\circ}, 60^{\circ}\right]$ over frequency. It was calculated for both polarisations from patterns determined with both GRASP and FEKO. The system temperature increases linearly from $8.2 \mathrm{~K}$ to $8.9 \mathrm{~K}$ across the frequency band. 
As the horn has no concentration of electric fields, the loss is small, in the order of $0.005 \mathrm{~dB}(\approx 0.4 \mathrm{~K}$ system noise contribution).

The mechanically robust OMT consists of a pair of orthogonal conical dipoles placed in the circular waveguide feeding the horn at approximately a quarter wavelength (at centre frequency) from a fixed backshort ${ }^{26}$. The balanced dipole excitations are converted to unbalanced coaxial transmission lines using folded Marchand baluns. The horn's reflection coefficient cannot be tuned to a negligible number, given the wide frequency range of the feed, and thus needs to be taken into account in the OMT optimisation. The EM model of the OMT and horn (i.e. feed) is computationally expensive and not suitable for optimisation. A methodology was devised that employed problem decomposition together with a combination of high fidelity EM and physics-based models to optimise the OMT ${ }^{27}$. The optimisation goal was to minimise the reflection coefficients of the coaxial transmission lines from the OMT and minimise the coupling between the signal channels. Several mechanical constraints were also taken into account. The resultant reflection coefficient of the feed is given in Figure 22.

Due to the OMT's compact structure and the minimisation of the dielectric material within the balun structures, the loss is low. The predicted loss is of the order of a quarter wavelength air-filled coaxial transmission line, i.e. less than $0.03 \mathrm{~dB}$ $(<0.6 \mathrm{~K}$ system noise contribution).

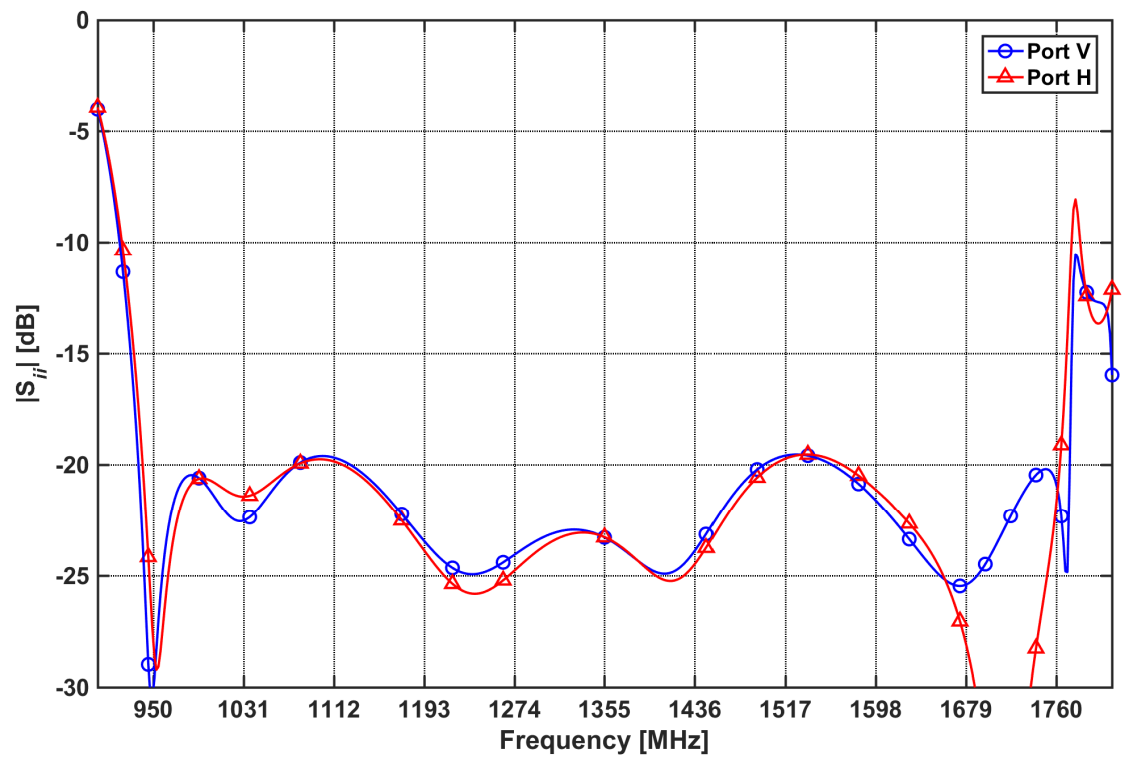

Figure 22. Predicted input reflection coefficients of both OMT ports with the OMT terminated in the feed horn.

A symmetric quarter wave directional coupler, with coupling level of $35 \mathrm{~dB}$, follows the OMT. This coupler can couple a known noise signal into both the signal paths for the purpose of receiver calibration. Again using air-filled transmission lines for the through lines the loss is minimised and is of the order of $0.03 \mathrm{~dB}$.

A short semi-rigid aluminium cable connects the signal paths from the directional coupler to the first stage low noise amplifiers (LNAs). These cables provide thermal isolation between the coupler/OMT and the cold (15 K) LNA. The cables' loss contribution is about $1 \mathrm{~K}$ to $1.5 \mathrm{~K}$. The LNA has a system noise contribution of less than $3 \mathrm{~K}$ and was designed by the Herzberg Institute of Astrophysics (HIA).

The feed's noise contribution to the system temperature is expected to increases from $6.2 \mathrm{~K}$ at the low frequency to $6.9 \mathrm{~K}$ at the high frequency.

\section{SKA1-MID RECEIVER / DIGITIZER}

The SKA1-Mid dish digitizer takes pre-amplified RF signal in two polarities from the selected feed and applies Analogue to Digital Conversion (ADC) to capture the science signal in a stream of digital samples. The samples undergo 
digital signal processing in a Field Programmable Gate Array (FPGA) and then formatted by the FPGA as network packets and shipped over the 100G Ethernet to the "Central Signal Processor" (CSP) facility. Before discretization by the $\mathrm{ADC}$ the RF signal must be amplified and band pass filtered to reject out-of-band frequencies and best utilize the ADC numerical range. At the minimal setting of variable attenuator the RF path gain must be sufficient for faint astronomy signals to fill the numerical range of ADC while stronger astronomical sources and terrestrial interferences must be digitized without ADC saturation at higher attenuation settings. This essential functionality is shown for the SKA1-Mid Band 1 in Figure 23.

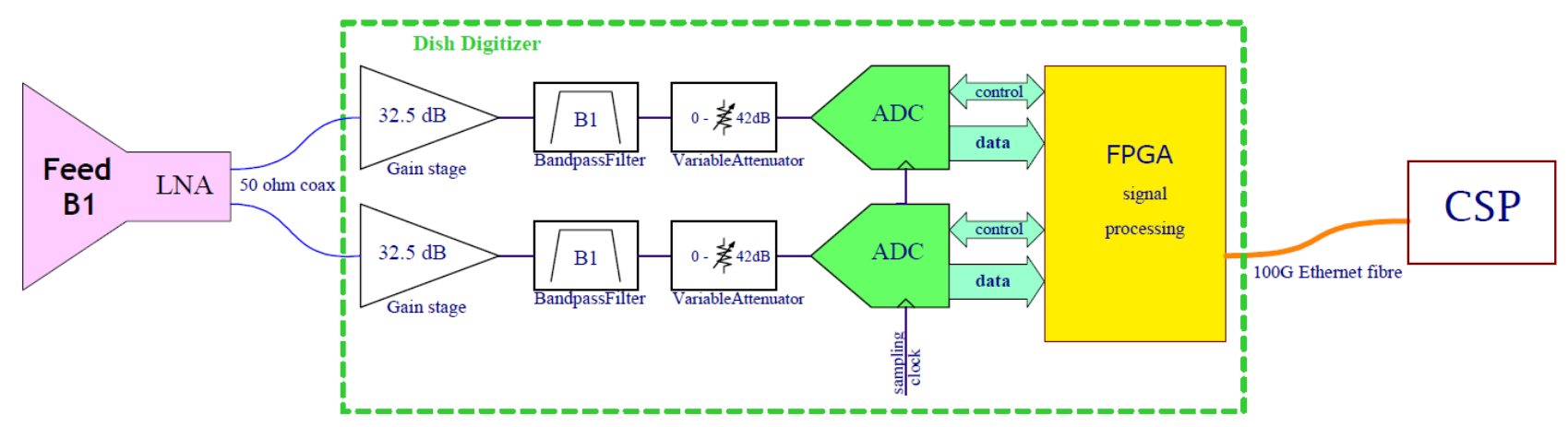

Figure 23. Dish Digitizer signal chain for Band 1.

The SKA1 Baseline Design ${ }^{5}$ specifies the bandwidths to be captured and digitization bit-depths for all SKA bands as summarized in Table 1. Bands 2, 3 and 4 have high-to-low frequency ratio of 1.85 which allows sampling in $2^{\text {nd }}$ Nyquist zone while Bands 1 and 5 have higher band ratios and must be sampled in $1^{\text {st }}$ Nyquist zone.

An additional Baseline Design requirement for Band 5 is that the digitizer must extract from the full bandwidth two selectable sub-bands of $2.5 \mathrm{GHz}$ and only ship these to the CSP.

Table 1. SKA1-Mid frequency bands.

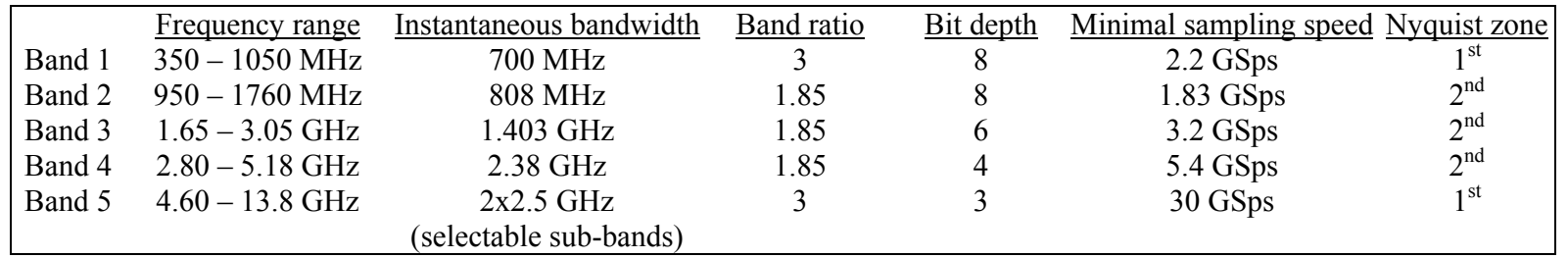

Since the ultra-fast ADC devices and FPGA signal processors are the most costly digitizer components it is desirable to share these among multiple bands. Given the frequency ranges of SKA1-Mid bands and bit depths that are available in commercial ADC devices it follows naturally to have Bands 1-3 sampled by an 8-bit resolution (or higher) ADC running at speeds under 4 GSps and separately Bands 4-5 by a 4-bit ADC with 30 GSps capability.

\subsection{Band 1+2+3 ADC and FPGA selection}

We have selected the ADC12J4000 from Texas Instruments as a shared ADC for Bands 1-3. It is a 4GSps 12-bit device with 3.2 GHz RF bandwidth and it fully satisfies the requirements of Bands 1-3. It transmits digital samples on JESD204B serial lanes which greatly simplifies the data interface to the FPGA and ensures error free data streaming. This interface has been fully supported with IP cores for high end devices from major FPGA vendors.

In addition to ADC data streaming the FPGA is also tasked with formatting the data for $100 \mathrm{G}$ Ethernet transport (packetizing). To comply with the SKA1-mid data backhaul standards the FPGA must be equipped with 28 Gbps transceivers to support the CAUI-4 electrical interface and must implement the IEEE 802.3bm-2015 network protocol. To meet these requirements we have selected the Xilinx Virtex Ultrascale XCVU095 as the development platform for Dish Digitizer.

A further simplification and cost reduction is realized by sharing the digital attenuators between Bands 1-3, resulting in the digitizer signal chain illustrated in Figure 24. 
Even though the deployment of SKA1-Mid Band 3 feed has been deferred to a later date, the capacity for Band 3 can be included in the initial configuration of SKA1-Mid digitizer with relatively small incremental cost of Band 3 specific filters and gain stages.

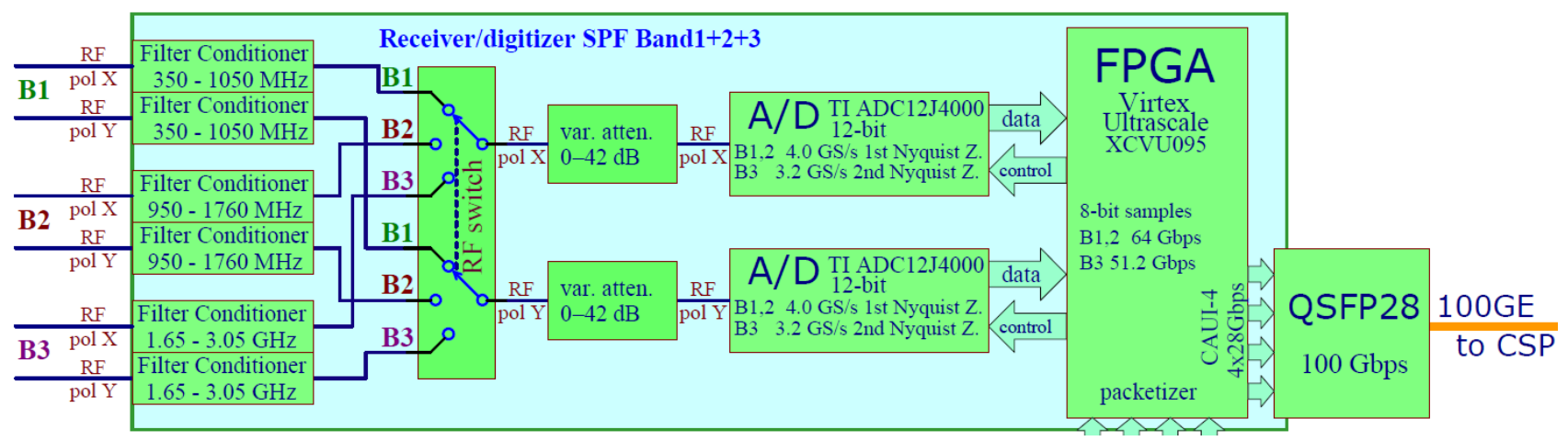

Figure 24. Band 1+2+3 digitizer based on shared TI ADC12J4000 analog to digital converter.

\subsection{Band 4+5 ADC selection}

Similarly to Band 1+2+3 digitizer, if a 4-bit $30 \mathrm{GSps}$ ADC is used as Band 5 digitizer it can also be shared with Band 4 and provide Band 4 sampling at a relatively small additional cost (the RF switch and Band 4 specific filters and amplifiers). Since there are no commercial ADC devices that meet the performance requirements for Band 5 we are developing a custom 2-way interleaved ADC, constructed from two commercial off the shelf 4-bit ADC devices operating at 15 GSps. Based on this custom ADC we will assemble a combined Band $4+5$ digitizer as illustrated in Figure 25. A dedicated high performance FPGA board carries out data streaming from ADC devices for two RF polarities and performs Digital Down-Conversion (DDC) to extract two tuneable sub-bands of $2.5 \mathrm{GHz}$ from the full digitized bandwidth of Band 5. The sub-bands are re-sampled by the DDC at 6 GSps in 4-bit resolution and the combined raw data stream of $96 \mathrm{Gbps}$ is passed on to the Band 123 FPGA for packetizing and 100GE transport to the CSP.

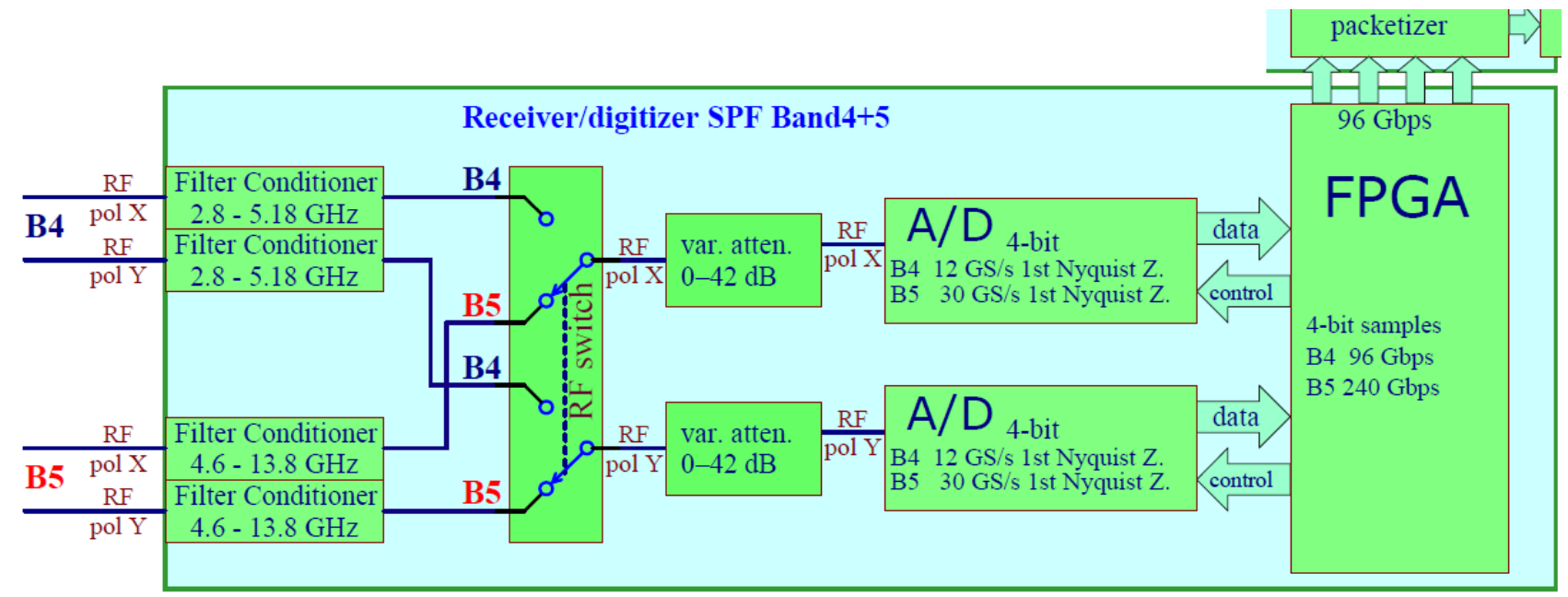

Figure 25. Band 4+5 digitizer based on shared 4-bit analog to digital converter.

\subsection{Digitizer circuits location}

The digitizer circuits location and housing must be considered carefully from the perspective of avoiding electromagnetic interference (EMI) both internal between digitizer components as well as external coupling from the digitizer to the extremely sensitive feeds of the host dish and adjacent dishes of the array. The location choice also 
directly impacts the digitizer performance, cost and maintainability. We have compared the four location alternatives collected in Table 2 in an internal SKA Dish Consortium trade-off study.

Table 2 Digitizer location options

\section{Location}

1) Complete Receiver system installed inside the pedestal with long RF co-ax lines connecting the Feeds to the Receivers through cable wraps and guides.

2) Complete Receiver system installed on the Feed Indexer ( $<10 \mathrm{~m}$ from Feeds). Only fibre optic links (clocks \& digital data) and power cables connecting to the pedestal.

3) Majority of Receiver system installed inside the pedestal, with RF over fibre links connecting to the ADC modules which are installed inside the pedestal. Power cables are required to support the $\mathrm{RF}$ over fibre and related modules.

4) All analogue signal path components including ADC's are installed on the Feed Indexer $(<10 \mathrm{~m}$ from Feeds), with digital fibre optical links connecting the ADC outputs to the digital processing modules. Digital processing modules and support systems are installed inside the pedestal. Power cables and clock lines (over fibre) are required for the active electronics.

The trade-off study recommended architecture 4 for implementation in the SKA1-Mid and Band $1+2+3$ as well as Band $4+5$ digitizers are being built in prototypes following this recommendation as illustrated in Figure 26.

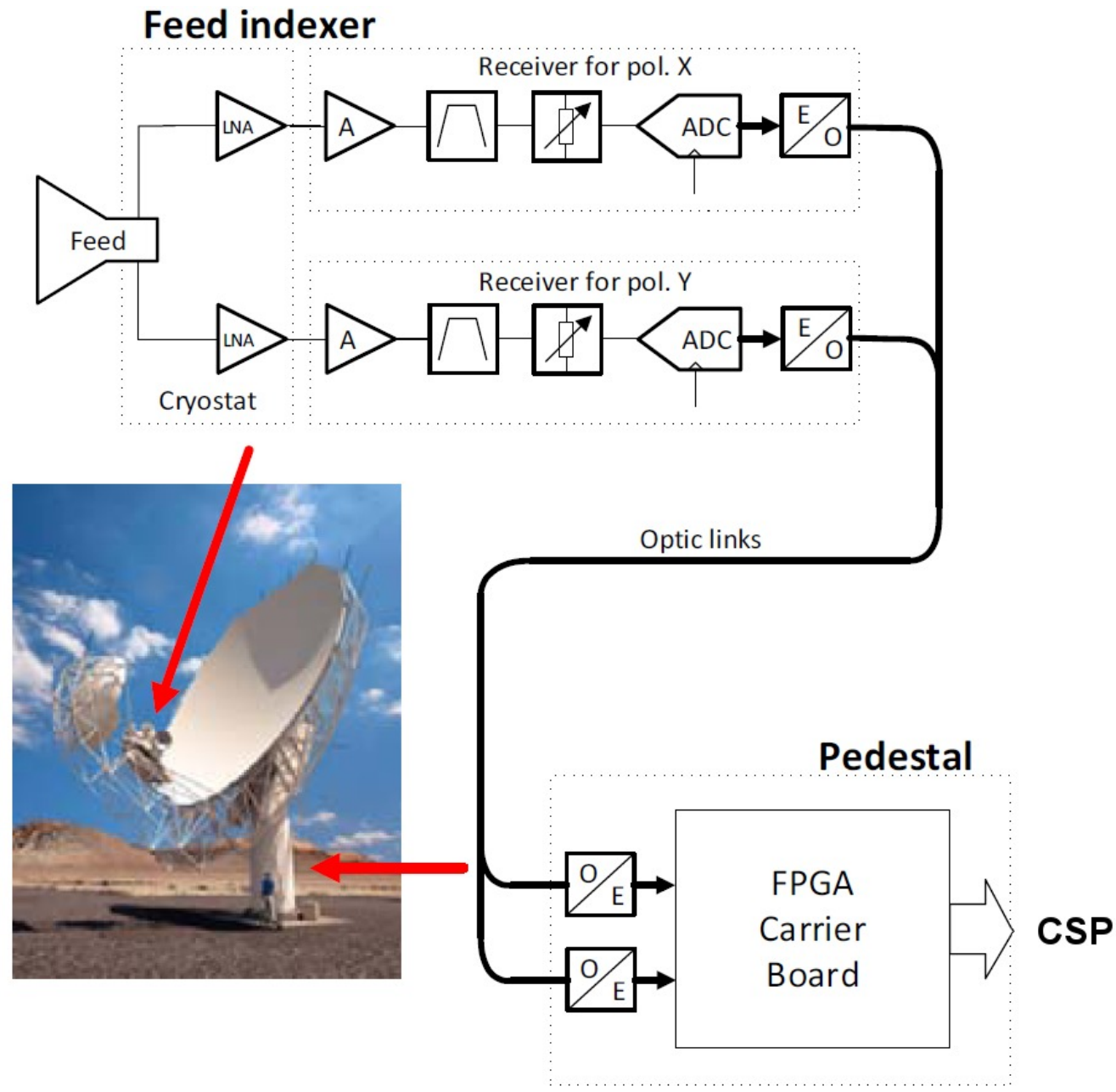

Figure 26. SKA1-Mid digitizer components location. 


\section{REFERENCES}

[1] “Square Kilometre Array”, SKA Organisation, https://www.skatelescope.org/ (2016).

[2] "Advancing Astrophysics with the Square Kilometre Array - Volume 1", Proc. AASKA, SKA Organisation, https://www.skatelescope.org/wp-content/uploads/2011/03/SKA-Astophysics-Vol1.pdf (2015).

[3] "Advancing Astrophysics with the Square Kilometre Array - Volume 2", Proc. AASKA, SKA Organisation, https://www.skatelescope.org/wp-content/uploads/2011/03/SKA-Astophysics-Vol2.pdf (2015).

[4] Braun, R., et al., "SKA1 Level 0 Science Requirements", SKA-TEL-SKO-0000007 Rev. 2, SKA Organisation (28 Oct. 2015).

[5] Dewdney, P.E., et al., "SKA1 System Baseline Design V2", SKA-TEL-SKO-0000002 Rev. 3, SKA Organisation (26 Feb. 2016).

[6] "South Africa's MeerKAT Radio Telescope: Technical Fact Sheet", SKA South Africa, http://www.ska.ac.za/download/fact_sheet_meerkat 2014.pdf (Mar. 2014).

[7] Theron, I. P., Lehmensiek, R., and de Villiers, D. I. L., "The design of the MeerKAT dish optics," Proc. Int. Conf. Electromagn. Adv. Appl. (ICEAA), Cape Town, South Africa, 539-542 (Sep. 2012).

[8] I. P. Theron, R. Lehmensiek and D. I. L de Villiers, "Towards an optics design for the SKA," Proc. IEEE Africon/URSI Conf., Mauritius, 1313-1317 (Sep. 2013).

[9] Lehmensiek, R., and de Villiers, D. I. L., "Sensitivity performance of the SKA offset Gregorian reflector candidates with ideal feeds," Proc. IEEE Int. Symp. AP \& USNC/URSI Nat. Radio Sci. Meet., Memphis, TN, 1479-1480 (July 2014).

[10] de Villiers, D. I. L., and Lehmensiek, R., "Sub-Reflector Extensions for Reduced Noise Temperature in LowSide Sub-Reflector Offset Gregorian Systems," Proc. $6^{\text {th }}$ Eur. Conf. Antennas and Propag. (EuCAP), Praque, Czech Republic, 3438-3441 (Mar. 2012).

[11] Medellín, G. C., “Antenna noise temperature calculation,” SKA Memo 95 (July 2007).

[12] Lehmensiek, R., Theron, I. P., and de Villiers, D. I. L., "Deriving an optimum mapping function for the SKA shaped offset Gregorian reflectors," IEEE Transactions on Antennas and Propagation, 63(11), 4658-4666 (Nov. 2015).

[13] Smith, S., and Hay, S., "Analysis of offset Gregorian reflector with phased array feed for SKA," Proc. IEEE Int. Symp. AP \& USNC/URSI Nat. Radio Sci. Meet., Vancouver, BC, Canada, 1522-1523 (July 2015).

[14] Lehmensiek, R., "SKA optics options: SPF EM analyses," SKA organisation, Internal project document (Dec. 2014).

[15] Billade, B., "Band 1 feed selection document, performance and cost study," SKA organisation, Internal project document (Oct. 2014).

[16] Akgiray, A., Weinreb, S., Imbriale, W., and Beaudoin, C., "Circular quadruple-ridged flared horn achieving near-constant beamwidth over multioctave bandwidth: Design and measurements," IEEE Transactions on Antennas and Propagation, 61(3), 1099-1108 (Mar. 2013).

[17] CST, “CST MICROWAVE STUDIO,” http://www.cst.com/Content/Products/MWS/Overview.aspx.

[18] Ivashina, M., Iupikov, O., Maaskant, R., van Cappellen, W., and Oosterloo, T., "An optimal beamforming strategy for wide-field surveys with phased-array-fed reflector antennas," IEEE Transactions on Antennas and Propagation, 59(6), 1864-1875 (June 2011).

[19] TICRA, "GRASP," http://www.ticra.com/products/software/grasp.

[20] Schleeh, J., et al., "10 K Room Temperature LNA for SKA Band 1", submitted: International microwave symposium, IMS2016 (2016).

[21] Lehmensiek, R., and Theron, I. P., "Minimizing the MeerKAT system noise temperature," $31^{\text {th }}$ URSI General Assembly and Scientific Symposium (GASS), Beijing, China, 1-4 (Aug. 2014).

[22] Lehmensiek, R., and de Villiers, D. I. L., "Wide Flare Angle Axially Corrugated Conical Horn Design for a Classical Offset Dual-Reflector Antenna," Proc. $6^{\text {th }}$ Eur. Conf. Antennas and Propag. (EuCAP), Praque, Czech Republic, 3292-3294 (Mar. 2012).

[23] Lehmensiek, R., and Theron, I. P., "The design of the MeerKAT UHF band feed," Proc. $8^{\text {th }}$ Eur. Conf. Antennas and Propag. (EuCAP), The Hague, Netherlands, 880-884 (Apr. 2014).

[24] Altair, FEKO, Suite 7.0, http://www.feko.info.

[25] Kildal, P-S., "Combined E- and H-plane phase centers of antenna feeds," IEEE Transactions on Antennas and Propagation, 31(1), 199-202 (Jan. 1983). 
[26] Lehmensiek, R., and Theron, I. P., "The design of the MeerKAT L-band feed," Proc. Int. Conf. Electromagn. Adv. Appl. (ICEAA), Cape Town, South Africa, 321-324 (Sep. 2012).

[27] Lehmensiek, R., "A design methodology of the wideband orthogonal mode transducer for the SKA band 2 feed," Proc. $10^{\text {th }}$ Eur. Conf. Antennas and Propag. (EuCAP), Davos, Switzerland, (Apr. 2016). 